\title{
Evaluation of a Hybrid Boltzmann-Continuum Method for High-Speed Nonequilibrium Flows
}

\author{
Jonathan M. Burt* and Eswar Josyula \\ U.S. Air Force Research Laboratory, Wright-Patterson Air Force Base, Ohio 45433 \\ and \\ Timothy R. Deschenes $\stackrel{\ddagger}{\sharp}$ and Iain D. Boyd표 \\ University of Michigan, Ann Arbor, Michigan 48109
}

\begin{abstract}
DOI: $\underline{10.2514 / 1.51406}$
A novel hybrid code for simulation of continuum and/or rarefied compressible gas flows is evaluated for a set of hypersonic blunt-body flow problems. This code, named the unified flow solver, combines several compressible gasflow simulation schemes for application to a wide range of Knudsen number regimes and features capabilities for strong coupling between low Knudsen number and high Knudsen number schemes along with automatic binary treebased grid adaptation. Unified flow solver simulations are performed for Mach 4 monatomic flows over a cylinder at various global Knudsen numbers, and simulation results are compared with results from simulations that employ direct simulation Monte Carlo and continuum Navier-Stokes solvers. Although good agreement is generally found, significant differences are identified and are used to highlight limitations or advantages of various simulation schemes in a unified flow solver. Results from this comparison are used to explain discrepancies in an earlier study involving application of similar techniques to a rarefied expansion flow.
\end{abstract}

\section{Introduction}

$\mathbf{T}$ HE effectiveness of intermolecular collisions in driving the distribution of gas molecule velocities toward equilibrium is typically quantified by the Knudsen number $K n$, which in turn is defined as the ratio of the mean free path to some characteristic length scale based on boundary geometry or gradients. A variety of gas-flow problems are characterized by a wide range of local Knudsen number regimes, with strong translational nonequilibrium in some portions of the flowfield and near-equilibrium velocity distributions in other flowfield regions. These problems include atmospheric flows around reentry vehicles, launch vehicles and hypersonic cruise vehicles; rocket exhaust flows at high altitudes; spacecraft gas venting; other hypersonic flows for which the internal shock structure is important; and subsonic flows, such as those within MEMS devices, which involve either very small length scales or low gas density.

For accurate simulation of high Knudsen number rarefied regions within these flows, translational nonequilibrium effects must be considered, and either a particle scheme such as the direct simulation Monte Carlo (DSMC) method [1] or a direct simulation method for the governing Boltzmann equation [2] is required. Although these methods can be applied to low Knudsen number regions as well, they tend to be far more computationally expensive than continuum computational fluid dynamics (CFD) methods based on the NavierStokes or Euler equations, while offering the same degree of accuracy in low Knudsen number regimes. Thus, an optimal combination of accuracy and efficiency for simulation of mixed rarefied-

Presented as Paper 2010-1569 at the 48th AIAA Aerospace Sciences Meeting, Orlando, FL, 4-7 January 2010; received 1 July 2010; revision received 1 April 2011; accepted for publication 8 April 2011. This material is declared a work of the U.S. Government and is not subject to copyright protection in the United States. Copies of this paper may be made for personal or internal use, on condition that the copier pay the $\$ 10.00$ per-copy fee to the Copyright Clearance Center, Inc., 222 Rosewood Drive, Danvers, MA 01923; include the code $0887-8722 / 11$ and $\$ 10.00$ in correspondence with the CCC.

${ }^{*}$ Currently Research Scientist, Universal Technology Corporation. Member AIAA.

${ }^{\dagger}$ Senior Research Aerospace Engineer. Associate Fellow AIAA.

${ }^{\ddagger}$ Graduate Student, Department of Aerospace Engineering. Student Member AIAA.

${ }^{\S}$ Professor, Department of Aerospace Engineering. Associate Fellow AIAA. continuum flows generally requires integration of rarefied and continuum schemes within a coupled numerical framework. In this type of hybrid approach, the simulation domain is divided into rarefied and continuum flow regions through evaluation of a continuum breakdown parameter. DSMC or Boltzmann solver calculations are performed in rarefied regions, a continuum CFD method is applied in continuum regions, and coupling routines are used to exchange conserved quantities or other flow information across continuum breakdown boundaries.

In recent years, most work on algorithm development for mixed rarefied-continuum flow simulations has focused on hybrid CFDDSMC techniques [3-8]. Such techniques have been shown to preserve the physical accuracy of DSMC in high Knudsen number regions, while offering the numerical efficiency of a CFD NavierStokes solver in low Knudsen number regions. However, the inherent statistical scatter in DSMC can make coupling between CFD and DSMC calculations difficult, and much of the focus in hybrid DSMC-CFD development has been on scatter reduction to avoid inaccurate or unstable CFD solutions. The scatter problem becomes particularly severe in simulations of highly unsteady flows, subsonic flows, or flow problems (such as those involving gas radiative emission or chemistry) for which accurate characterization is desired for the low-probability tails of the velocity distribution. In these cases, a very large number of DSMC particles may be required for sufficient scatter reduction, and a hybrid CFD-DSMC simulation can be prohibitively expensive.

In a recently proposed alternate scheme by $\mathrm{Xu}$ and Huang [9] for multiscale simulation of mixed rarefied-continuum flows, the Bhatnagar-Gross-Krook (BGK) approximation of the Boltzmann equation [2] is solved in discretized velocity space without operatorsplitting between collision and advection processes. The standard BGK operator-splitting approach limits the allowable time-step size to the order of the mean collision time, which can make BGK simulation of low Knudsen number flows prohibitively expensive. In the scheme of $\mathrm{Xu}$ and Huang [9], however, collisional modifications are imposed on fluxes across cell interfaces, without any approximations of decoupled collision and advection processes over the time-step interval. As a result, practical calculations for multiscale flows can be performed by means of a single technique in both rarefied and continuum regions. Despite significant potential, this scheme suffers from reduced efficiency in continuum regions relative to traditional Navier-Stokes schemes, for which only a small number 
of conserved quantities, not the probability density at a large number of velocity space nodes, are calculated.

Another approach for simulation of mixed rarefied-continuum flows involves coupling DSMC to a particle method intended for continuum flow simulation. One promising scheme of this type is that of Burt and Boyd [10,11], which incorporates a low diffusion particle method to minimize numerical diffusion and dissipation effects typically associated with low Knudsen number particle simulations. The particle-particle hybrid approach benefits from simplified information exchange along continuum breakdown boundaries, but as with the scheme of Xu and Huang [9], this type of approach tends to suffer from relatively low efficiency in continuum flow calculations.

In another alternative hybrid scheme, a set of direct simulation methods for the Boltzmann equation are coupled to kinetic methods for the compressible Navier-Stokes or Euler equations [12-16]. This hybrid scheme, implemented in the unified flow solver (UFS) code, is not prone to the DSMC statistical scatter problem that may be considered the main drawback in CFD-DSMC techniques. Instead of using a collection of particles to represent the gas velocity distribution within rarefied regions, as in DSMC, nonequilibrium velocity distributions in UFS are modeled using a uniform Cartesian grid in velocity space. Discrete approximations of the advection and collision terms in the Boltzmann equation are employed to update the probability density at grid points in both velocity and physical space, and coupling across continuum breakdown boundaries is performed through two-way information exchange during each simulation time step.

The UFS code is based on the open source framework of the Gerris flow solver [17] and includes capabilities for binary tree-based Cartesian grid adaptation. Solid two-dimensional or three-dimensional objects are easily integrated within the simulated flowfield through the use of cut-cell boundaries, and additional procedures allow optimized load balancing for parallel domain decomposition. Continuum breakdown evaluations are periodically performed during a UFS simulation, for automatic assignment of each grid cell to either continuum or rarefied domains. Performance improvements, relative to DSMC or full Boltzmann solutions, are realized in UFS through the use of continuum CFD methods where appropriate and through initialization of rarefied domain calculations with (at least partially converged) continuum method solutions. In comparison with DSMC, reduced simulation expense has been demonstrated in UFS simulations of a nozzle/plume expansion flow [14], and comparable expense to DSMC has been observed in UFS simulations of a hypersonic blunt-body flow [15].

In the current effort, UFS is applied to a set of high-Mach-number rarefied/continuum flows over a cylinder and is evaluated for accuracy and efficiency through comparison with DSMC and CFD Navier-Stokes methods. Although previous evaluations of UFS have recently been carried out for a variety of flow problems, new objectives here include performing a more comprehensive comparison for accuracy and efficiency with both DSMC and CFD for a representative set of hypersonic blunt-body flows, and highlighting limitations and relative advantages of the various schemes employed in UFS. As an additional motivation for this study, results are used to provide some insight regarding the source of discrepancies between DSMC and UFS hybrid simulation results that were observed in previous work [14].

In the following sections, numerical approaches used in this study are outlined, and simulation setup and input parameters are described. Detailed simulation results are then presented. The accuracy of UFS simulation results is assessed by comparing surface flux coefficients, contour plots and the variation along the stagnation streamline in selected flow properties. Next, drag coefficients computed from these simulations are plotted as a function of global Knudsen number, in order to further assess accuracy of UFS simulations, compare with available experimental data, and evaluate the dependence of drag on the cylinder surface temperature. Results are used to help explain discrepancies in a previously published study involving UFS. Considerations of computational expense are then discussed, and general efficiency comparisons are made between different simulations and techniques employed in this study. Finally, results are summarized and various characteristics, error sources and limitations of simulation techniques in UFS are discussed.

\section{Numerical Approaches}

A brief overview is provided for several numerical schemes used in this study. Detailed descriptions of these schemes are included in the references, and the reader is referred to these references for further details.

For simulation of rarefied flows or nonequilibrium regions in a mixed continuum-rarefied flowfield, UFS employs numerical solutions to the governing Boltzmann equation on a uniform Cartesian grid in velocity space $[2,12]$. An explicit solution method for the full Boltzmann equation, with finite time-step intervals, discrete nodes in velocity space and finite volume cells in physical space, is available in UFS with either first- or second-order spatial accuracy. This method makes use of temporal decoupling between advection and collision terms in the Boltzmann equation,

$$
\frac{\partial f}{\partial t}+\nabla_{r} \cdot(\xi)=I(f)
$$

where $\boldsymbol{\xi}$ is a point in velocity space, $\boldsymbol{r}$ is a point in physical space, and $f(\boldsymbol{\xi}, \boldsymbol{r})$ is the local number density in six-dimensional phase space. Linear hyperbolic advection terms on the left side of this equation are considered using standard upwind transport procedures for each velocity space node, and the nonlinear integral term represented by the collision operator $I(f)$ is solved by means of a large number of simulated binary collisions. Velocity space nodes are selected to participate in collisions using low-discrepancy Korobov sequences [12], in order to speed statistical convergence relative to standard Monte Carlo integration techniques.

To avoid expensive evaluations of the collision term in Eq. (1) and to reduce the required number of velocity space nodes, UFS allows the option of solving the BGK approximation of the Boltzmann equation in either two or three-dimensional discretized velocity space [2]. The BGK equation uses a linear relaxation term in place of the nonlinear collision operator $I(f)$ and may be solved deterministically at much lower expense.

The kinetic Navier-Stokes (NS) solver of Xu [18] can be used in UFS for calculations within continuum flowfield regions, where $f$ approaches the low Knudsen number Maxwellian limit and where explicit consideration of the velocity distribution function is unnecessary for accurate simulation. This scheme uses analytical solutions to the BGK equation in calculating fluxes between neighboring finite volume cells and allows consistent kinetic representation of the entire flowfield when coupled to Boltzmann or BGK schemes in a hybrid UFS simulation.

In hybrid UFS simulations performed for this study, a continuum breakdown parameter based on characteristic length scales for velocity and pressure gradients is periodically evaluated throughout the computational grid, in order to assign flowfield regions to either continuum or rarefied domains. Rarefied regions are identified by comparing values of a breakdown parameter $S_{\mathrm{NS}}$ to a threshold value of 0.1 . Following [12], the breakdown parameter is defined as

$$
\begin{aligned}
& S_{\mathrm{NS}} \\
& =\lambda \sqrt{\left(\frac{\nabla p}{p}\right)^{2}+\left[\left(\frac{\partial u}{\partial x}\right)^{2}+\left(\frac{\partial v}{\partial y}\right)^{2}+\left(\frac{\partial w}{\partial z}\right)^{2}\right] /\left(u^{2}+v^{2}+w^{2}\right)}
\end{aligned}
$$

where $\lambda$ is the mean free path, $p$ is the pressure, and $(u, v, w)$ is the bulk velocity. UFS calculations in the rarefied domain use either Boltzmann or BGK schemes, and Navier-Stokes calculations are performed within continuum regions. Strong coupling between continuum and rarefied flow calculations is employed through twoway exchange of conserved quantities across continuum breakdown boundaries. In particular, the mass, momentum and energy density in 
Navier-Stokes cells that border continuum breakdown boundaries are employed to compute the flux into each velocity space node within neighboring Boltzmann/BGK cells, using a near-equilibrium flux approximation. Likewise, fluxes from Boltzmann/BGK cells into neighboring Navier-Stokes cells are calculated through a discrete velocity approximation for unidirectional moments of the local velocity distribution. More discussion of coupling procedures is provided in $[12,13]$.

For comparison with UFS simulation results, additional simulations are run using the DSMC method [1]. As mentioned above, DSMC is a probabilistic method that uses a large collection of particles (each representing a large number of atoms or molecules in the simulated flow) to reproduce the underlying physics of the Boltzmann equation. DSMC calculations avoid the complexity and difficulty of conservatively solving the nonlinear Boltzmann collision term on a discrete velocity grid. Binary collisions are represented in DSMC by conservative velocity resampling among probabilistically selected particle pairs, and advection terms in the Boltzmann equation are replicated by particle movement operations. During each time step in a DSMC simulation, separate procedures are employed for binary collisions, particle movement, the creation of new particles along inflow boundaries, and time-averaged sampling for field and surface flux quantities of interest.

\section{Simulation Setup}

As the basis for a series of test problems, we consider a flow of argon over a cylinder with a freestream Mach number of 4 . We employ the hard-sphere collision model, for which collision cross sections are independent of the relative speed between colliding particles and for which viscosity varies as the square root of temperature [1]. Note that the hard-sphere model is used to determine collision cross sections only in Boltzmann and DSMC calculations; intermolecular collisions are not directly modeled in BGK and NS simulations, and hard-sphere approximations are instead used to determine the BGK collisional relaxation rate and the NS transport coefficients. Diffuse reflection with full thermal accommodation to the wall temperature is assumed on the cylinder surface, and the temperature at the wall is set to 1.2 times the freestream temperature. The diffuse reflection boundary condition is modeled in UFS Boltzmann and BGK calculations by setting the reflected mass flux from the wall to equal the incident mass flux for each velocity grid node in all wall boundary cells and by setting the corresponding reflected momentum and energy fluxes equal to those expected for a quiescent equilibrium reservoir at the wall temperature. The same boundary condition is modeled in DSMC by means of probabilistic postcollision velocity assignment following any particle-wall collisions. In simulations for which NS calculations are used over the entire flowfield, a no-slip wall boundary condition is employed. Simulations are performed for global Knudsen numbers, based on cylinder diameter, of $0.3,0.03$ and 0.003 using various models in UFS. For comparison, additional simulations are performed using the DSMC code MONACO [19] and the NS CFD code LeMANS [20]. Note that LeMANS simulations use the same hard-sphere transport coefficient approximations employed in the UFS NS calculations.

The $K n=0.3$ case is representative of a highly rarefied flow and is simulated using numerical solutions to the Boltzmann equation and the BGK equation [2]. A NS simulation of this flow is also performed using LeMANS. In the $K n=0.03$ case, large portions of the flowfield are within both rarefied and continuum regimes, and hybrid schemes involving coupled rarefied and continuum flow calculations should give the best combination of accuracy and efficiency. UFS simulations for this second case employ the following different options: BGK, NS, hybrid BGK-NS, and hybrid BGK-Euler. The accuracy of all UFS results at $K n=0.3$ and 0.03 is assessed through comparison with results from simulations using the DSMC code MONACO. The $K n=0.003$ case, for which only very small portions of the flowfield experience significant thermal nonequilibrium, is used to independently evaluate the NS solver in UFS by comparing UFS NS results with those from a NS simulation using the
LeMANS CFD code. To demonstrate the expected inaccuracy for NS simulations at $K n=0.3$ and 0.03 , LeMANS results are also generated for these cases.

MONACO simulations in this study employ unstructured grids that are automatically adapted to the local mean free path using an independent grid-generation utility, and dynamically adaptive DSMC subcells are used to ensure sufficiently small mean collision separation values. Spatially uniform time-step intervals in MONACO are carefully set to meet DSMC requirements based on the minimum mean collision time [1]. Numerical weight values are dynamically adapted in each cell to follow standard DSMC guidelines of at least 20 particles per cell, while avoiding any efficiency reduction due to excessive particle populations. LeMANS simulations use a finite volume implicit second-order modified version of the Steger-Warming flux vector splitting scheme [21], which is less dissipative in boundary layers but switches back to the original form in the vicinity of strong shocks.

Spatial and velocity grid independence and solution convergence is verified for all simulations. The full Boltzmann calculation uses a velocity grid of $40 \times 40 \times 20$ points, in the $x, y$, and $z$ directions, respectively, and BGK calculations are performed on a grid of $40 \times 40 \times 2$ points in velocity space. Both Boltzmann and BGK simulations take advantage of velocity distribution symmetry along the $z$ axis, in order to reduce the number of nodes in the velocity space grid. Boltzmann collision operations involve 10 simulated collisions per velocity grid node per iteration, as set by default in UFS in order to achieve a reasonable balance between accuracy and efficiency. Locally adaptive time-step intervals are employed in all UFS simulations, based on collisional relaxation times and a CourantFriedrichs-Lewy criterion [12]. The UFS Boltzmann simulation is performed on 16 processors in the nyx cluster at the University of Michigan, and all other UFS, MONACO, and LeMANS simulations are run using eight processors in the same cluster. The nyx cluster is composed of dual-core AMD Opteron CPUs, with a minimum of 2 Gbytes of memory and two processors per node. High parallel efficiency has previously been demonstrated for UFS, MONACO, and LeMANS on large numbers of processors, so code-specific differences in parallel scalability are assumed to have a relatively small effect on computational expense for all simulations presented here.

\section{Comparison of Simulation Results for $K n=0.3$}

Figure 1 shows computational grids used in UFS BGK and MONACODSMC simulations for the $K n=0.3$ case. Note that grids used in UFS calculations are adapted over the course of a simulation, and the BGK grid shown in Fig. 1 is taken from steady-state results after a total of 10,000 iterations. Ass observed in the figure, cell sizes in both the freestream and far-field wake regions are similar in the two simulations, although significant differences are found in cell

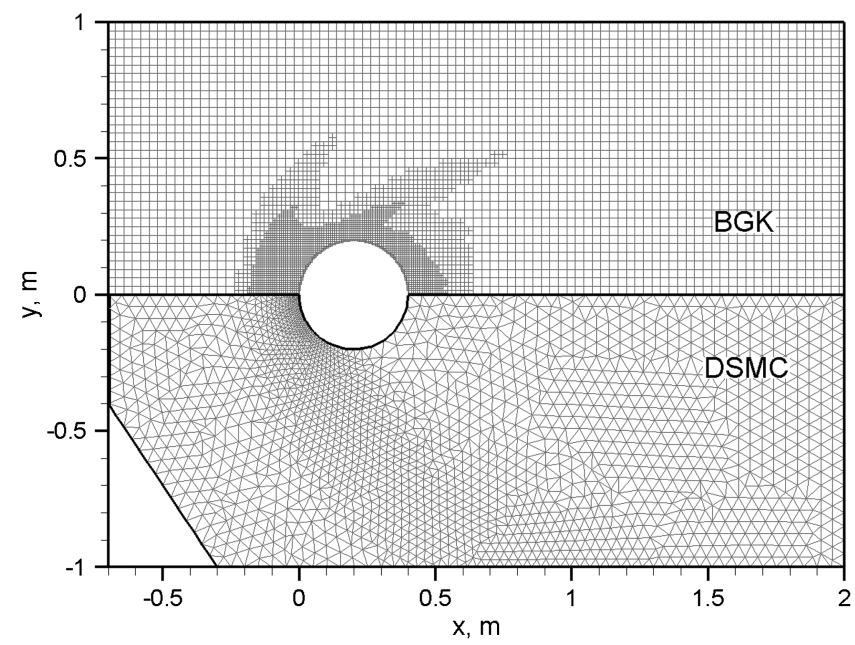

Fig. 1 Meshes for UFS BGK and DSMC simulations at $K n=0.3$. 


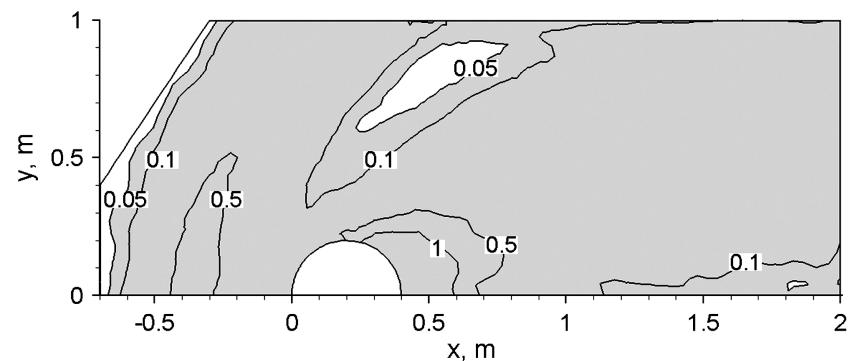

Fig. 2 Contours of the maximum-gradient-length local Knudsen number from the DSMC simulation at $K n=0.3$. Shaded area indicates regions of continuum breakdown.

size within shock layer and near-field regions. These discrepancies in cell size can be attributed to differences in grid refinement criteria between MONACO and UFS: MONACO cells are refined to the local mean free path, and grid refinement in UFS is based on density and velocity gradients.

In Fig. 2, contour lines are shown for the maximum-gradientlength local Knudsen number $K n_{\mathrm{GLL}-\max }$, as calculated from DSMC simulation results at $K n=0.3$. The parameter $K n_{\mathrm{GLL}-\max }$ is computed as the maximum ratio of the local mean free path $\lambda$ to length scales based on gradients in bulk velocity magnitude $V$, density $\rho$, or temperature $T$ :

$$
K n_{\text {GLL- max }}=\max \left\{\frac{\lambda}{a}|\nabla V|, \frac{\lambda}{\rho}|\nabla \rho|, \frac{\lambda}{T}|\nabla T|\right\}
$$

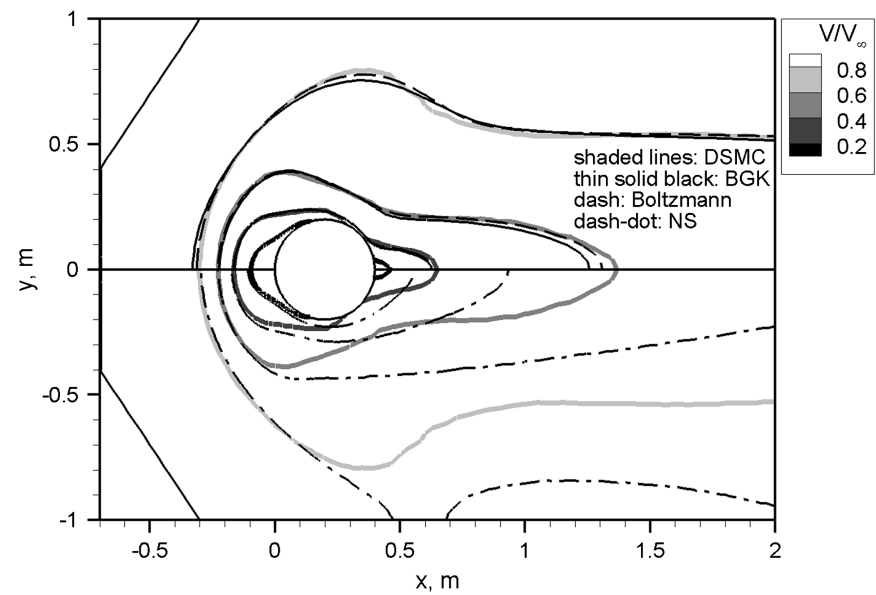

a)

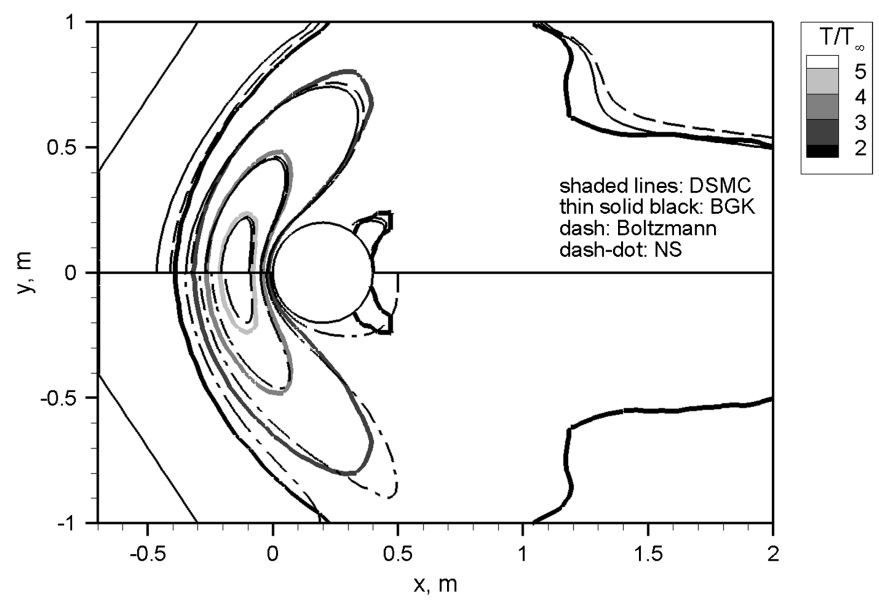

c)

Fig. 3 Field property contours for $K n=0.3$ : a) bulk velocity magnitude, b) density, and c) temperature. BGK and Boltzmann simulations are both run on UFS, the NS simulation is performed using LeMANS, and the DSMC simulation is run on MONACO.

where $a$ is the local speed of sound. The NS equations are usually assumed valid for $K n_{\mathrm{GLL}-\max }<0.05$ [22], and areas for which this condition is satisfied are shaded in Fig. 2. As shown in the figure, continuum breakdown occurs over nearly the entire flowfield.

Figure $3 \mathrm{a}$ shows contours of bulk velocity magnitude from MONACO, UFS and LeMANS simulations at $K n=0.3$. All values in the legend are normalized by the freestream velocity. Results from Boltzmann, BGK and DSMC simulations are shown in the upper half of the figure, and LeMANS NS and DSMC simulation results are compared in the lower half. As expected, both Boltzmann and BGK solutions show good overall agreement with DSMC, whereas the BGK results give slightly worse agreement with DSMC than Boltzmann simulation results. Poor agreement is observed between DSMC and NS results through much of the flowfield, which is consistent with the high level of continuum breakdown found in Fig. 2. Much of the discrepancy between DSMC and NS results can be attributed to the failure of assumptions underlying the NavierStokes equations; in regions of significant nonequilibrium, as indicated in Fig. 2, gradient-based diffusive transport approximations tend to break down, and the gas velocity distribution function cannot be accurately approximated using small perturbations from equilibrium. Both gradient transport and small perturbation assumptions are used in the NS calculations. The lack of velocity slip and temperature jump boundary conditions in the NS calculations also likely contribute to the disagreement between DSMC and NS results in Fig. 3a, although a nonslip boundary condition cannot by itself explain inaccuracy in a NS solution when strong rarefaction effects are present.

Figures $3 \mathrm{~b}$ and $3 \mathrm{c}$ show contours of normalized density and temperature, respectively, for all simulations at $K n=0.3$. Trends in

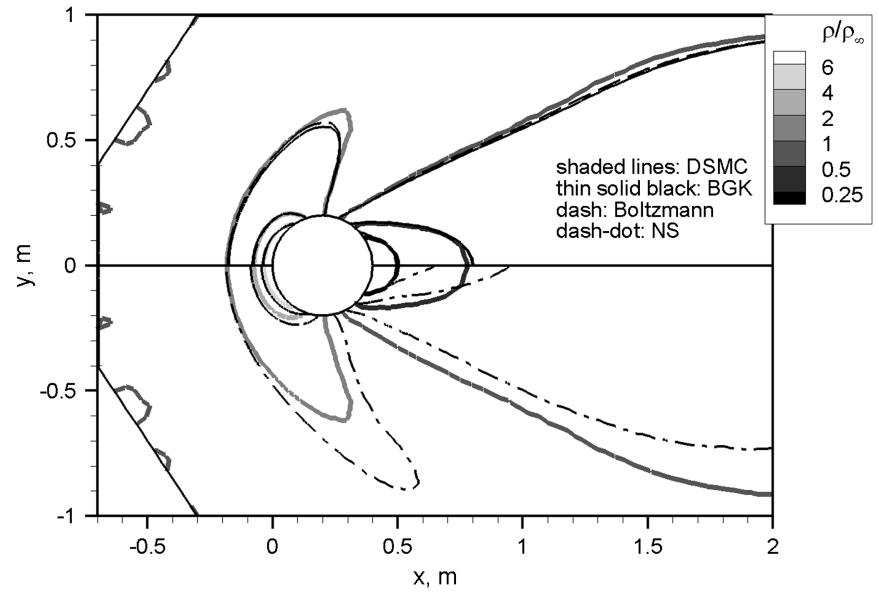

b) 
both figures are similar to those in Fig. 3a. In particular, good overall agreement is found between DSMC and Boltzmann simulation results, with only slightly worse agreement between DSMC and BGK. A significantly broader bow shock region, as indicated by temperature contours, is observed in BGK results than in results from either DSMC or Boltzmann simulations. As in Fig. 3a, poor agreement is found between DSMC and NS results over nearly the entire flowfield in both Figs. $3 \mathrm{~b}$ and $3 \mathrm{c}$.

Figures 4 show the variation along the stagnation streamline in the normalized bulk velocity, density and temperature from the $K n=$ 0.3 simulations. In all three figures we find very good agreement between Boltzmann and DSMC results, with only a small overestimate relative to DSMC in the shock thickness based on either velocity or temperature. Note that, as expected at this high global Knudsen number, the shock is represented in all simulations as a broad region of smooth compression, and the shock, shock layer, and boundary layer cannot be easily distinguished. Noticeable differences are observed, however, between DSMC, BGK and NS results in the level of diffusive transport: a significantly wider shock region is

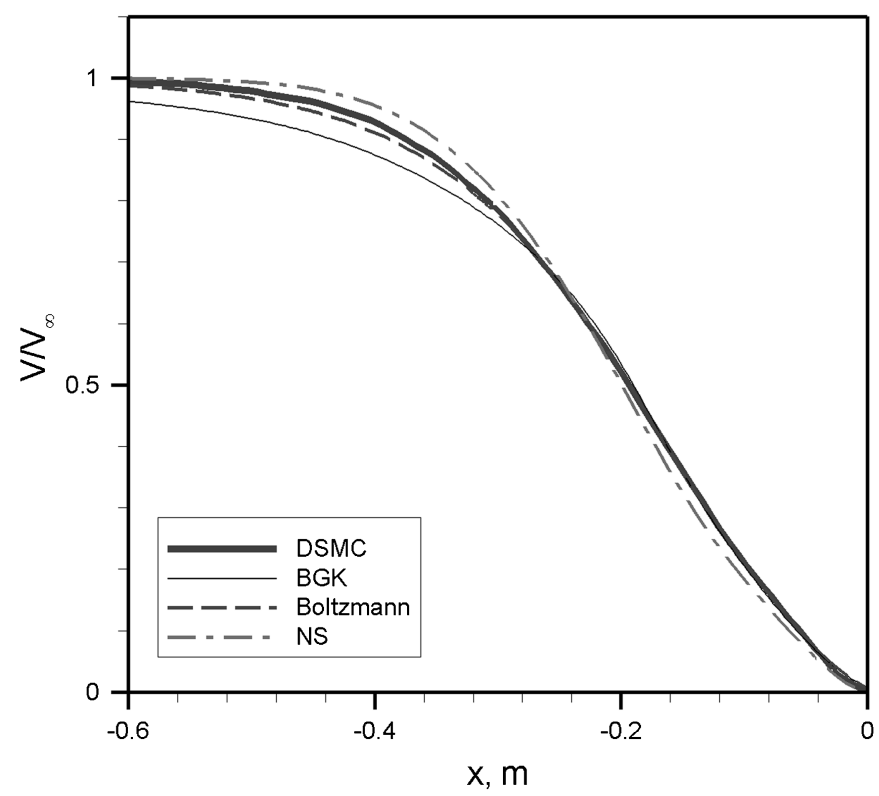

a)

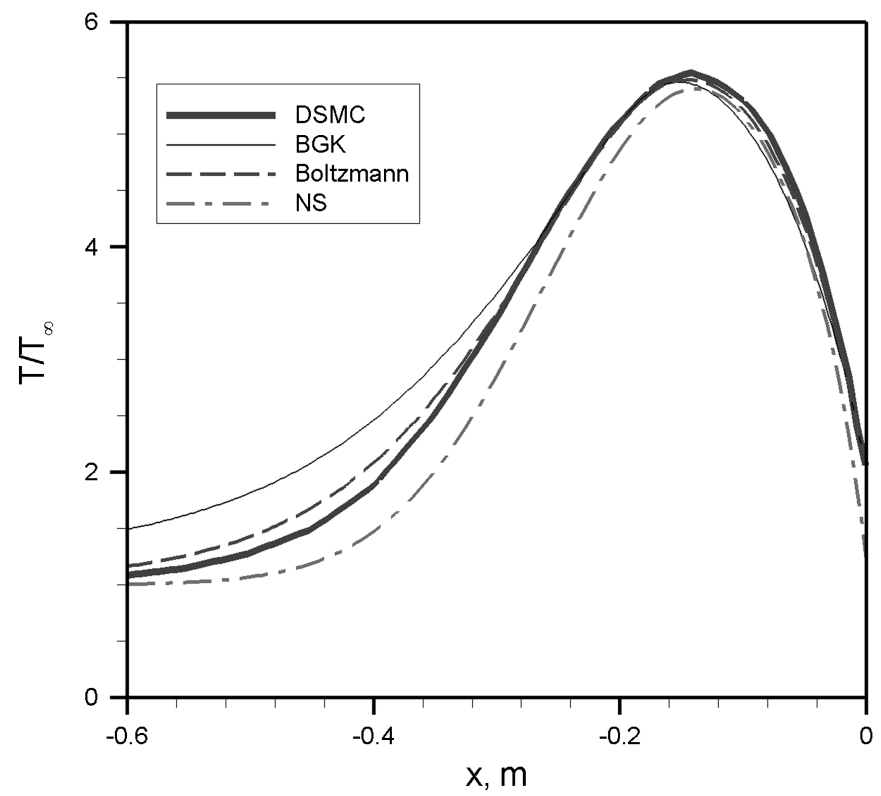

c) 


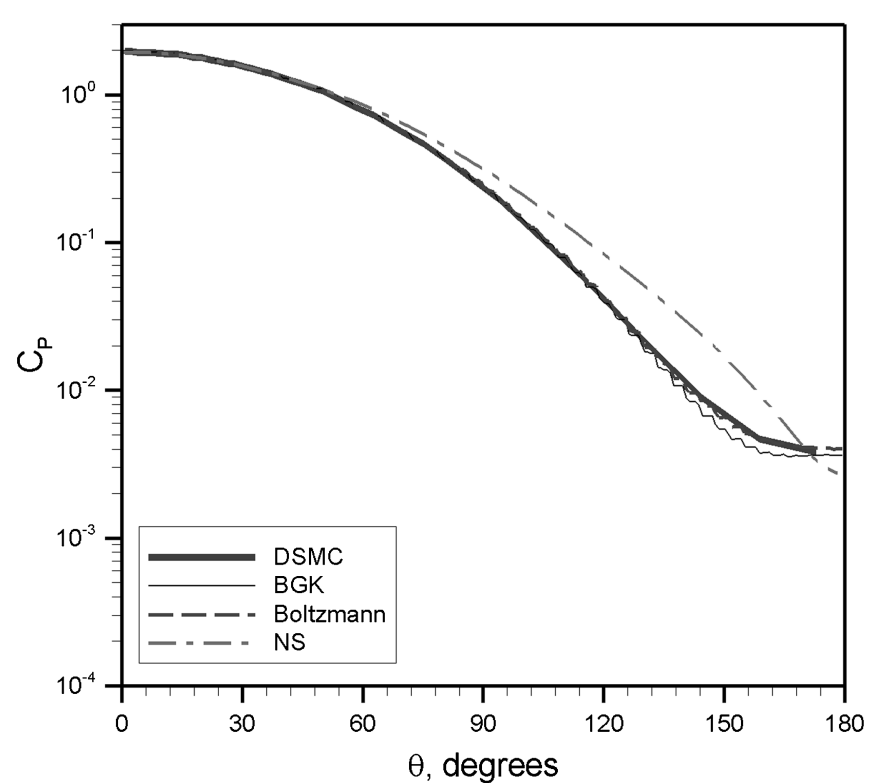

a)

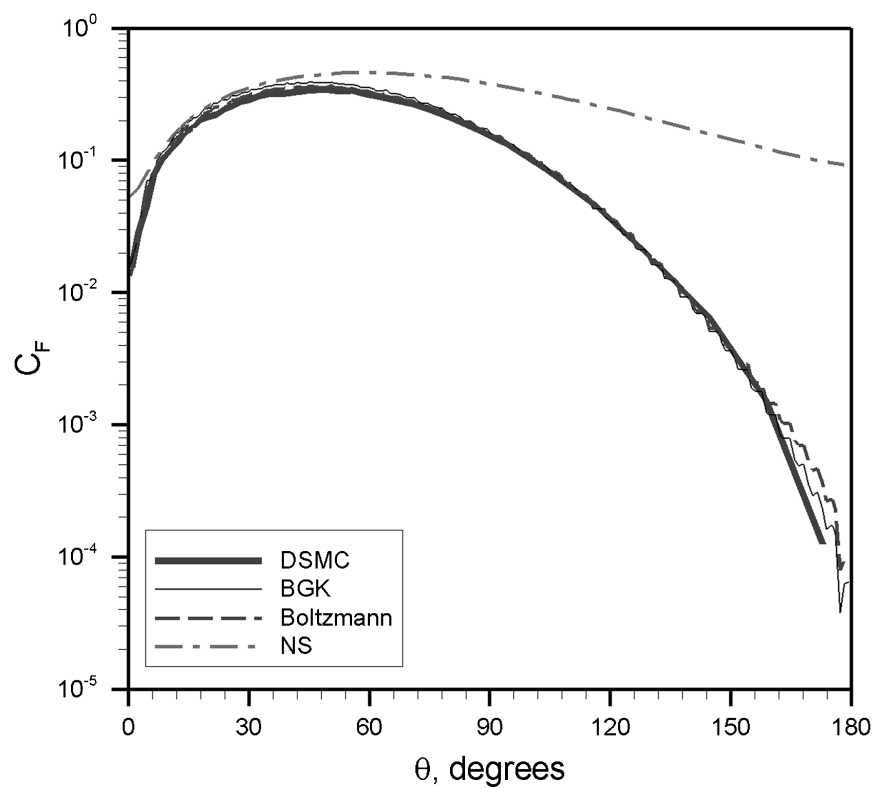

b)

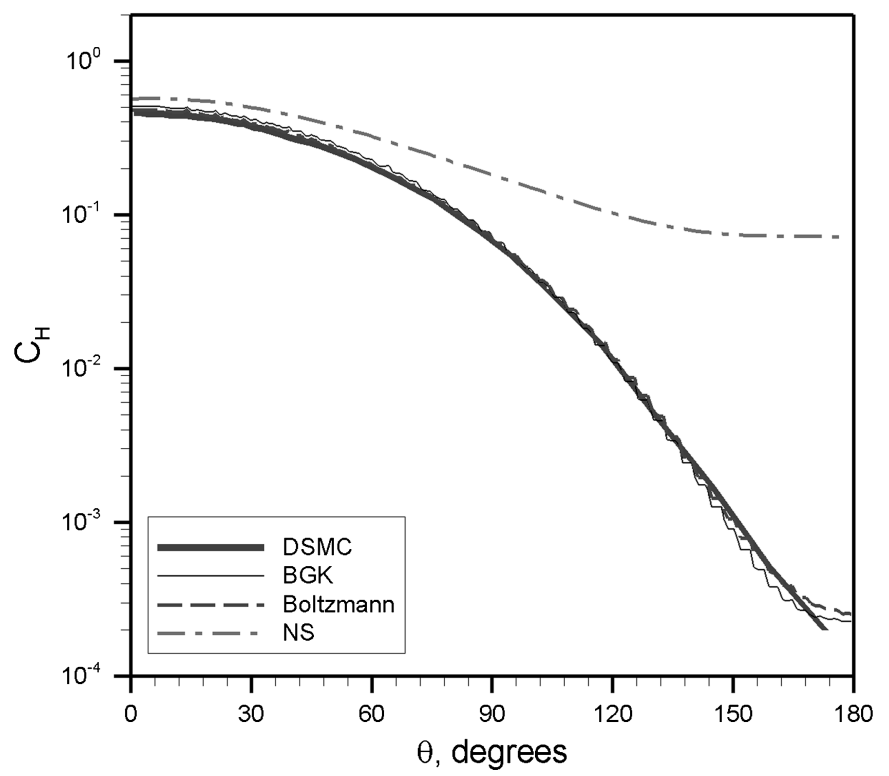

c)

Fig. 5 Surface flux coefficients for $K n=0.3$ : a) pressure, b) friction, and c) heat transfer. UFS is used for BGK and Boltzmann simulations.

figure, very good agreement is found between DSMC, BGK and Boltzmann results over the entire surface, whereas the friction coefficient is overestimated in the NS simulation by approximately a factor of 3 at $0^{\circ}$ and by over three orders of magnitude at $180^{\circ}$.

Figure $5 \mathrm{c}$ shows the surface heat transfer coefficient for the $K n=0.3$ simulations. Although surprisingly good agreement (within about 20\%) is found between NS and DSMC results at the front stagnation point, the level of disagreement increases rapidly with $\theta$, and the NS surface heat transfer is overestimated by around three orders of magnitude at $\theta=180^{\circ}$. Note that somewhat better agreement between NS and DSMC would be expected in Fig. 5c, as well as in Figs. 5a and 5b, if the NS wall boundary condition included consideration of velocity slip and temperature jump effects.

As in Figs. 5a and 5b, good overall agreement is found in Fig. 5c over the entire cylinder surface between DSMC, BGK and Boltzmann simulation results. Slightly larger discrepancies are found between DSMC and BGK than between DSMC and Boltzmann results, with a noticeable underestimate in BGK surface heat transfer along much of the afterbody and a small corresponding overestimate along the forebody. Although recently published UFS results have shown an overestimate in UFS heat transfer by approximately a factor of 2 [23], the authors were made aware of a normalization factor of 0.5 in UFS nondimensional heat transfer values that has been applied to values in Fig. $5 \mathrm{c}$ and that seems to provide far better agreement with DSMC. With this normalization factor, the overestimate relative to DSMC in stagnation point heat flux is only about $3.8 \%$ for the Boltzmann simulation and $9.5 \%$ for the BGK simulation.

Some of the error observed in Boltzmann and BGK values, as well as the artificial stair-step pattern in these values, is likely due to the cut-cell boundary condition and is expected to be eliminated through the use of the immersed boundary method in place of cut cells. Further error in surface heat transfer from Boltzmann and BGK simulations may be due to insufficient mesh refinement near the surface, although comparable cell sizes are used in these simulations as in the DSMC simulation and it seems unlikely that mesh resolution accounts for most of the discrepancy.

Another important contributor to the error in BGK results shown in Fig. 5c is the lack of a Prandtl number correction in BGK calculations. The BGK approximation of the Boltzmann equation gives transport coefficients that are functions of the collision frequency, but that lack the complex dependence on collision dynamics 


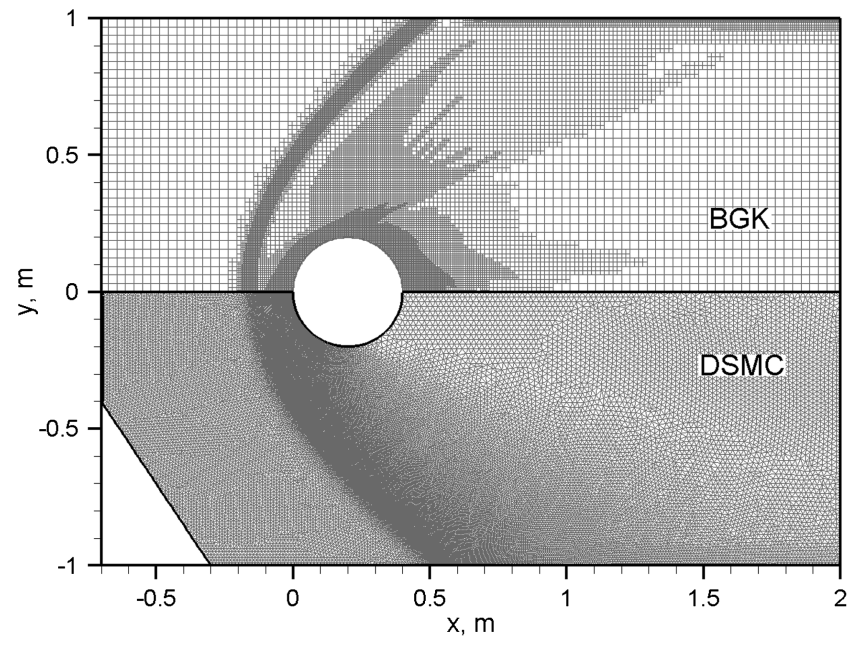

Fig. 6 Meshes for UFS BGK and MONACO DSMC simulations at $\mathrm{Kn}=\mathbf{0 . 0 3}$.

parameters for transport coefficients derived from the full Boltzmann equation. In particular, in the BGK equation the thermal conductivity cannot be specified independently from the viscosity, which results in a fixed Prandtl number of one. Various modifications of the BGK equation, such as the ellipsoidal statistical BGK equation, have been developed in order to overcome this limitation, and a Prandtl number correction has been implemented in a more recent version of UFS, which was not available for the present study. However, the BGK simulation results presented here should be understood to include errors associated with an underestimate in the gas thermal conductivity. More generally, an unphysical linearization of the collision term in the BGK approximation of the Boltzmann equation should correspond to some accuracy reduction in BGK calculation results.

\section{Comparison of Simulation Results for $K n=0.03$}

Figure 6 shows meshes used in UFS BGK and DSMC simulations for the $K \bar{n}=0.03$ case. As in Fig. 1, differences in local refinement levels are attributed to the fact that $\overline{D S M C}$ cell size is adapted to the mean free path, whereas UFS mesh adaptation is based on density and velocity gradients. For both simulations, the shock position is clearly indicated by a sudden increase in mesh refinement levels upstream of the cylinder.

Figure $\underline{7}$ is a contour plot of the maximum-gradient-length local Knudsen number $K n_{\mathrm{GLL}-\max }$ from the DSMC simulation at $K n=0.03$. In comparing this figure with Fig. 2, we find that continuum breakdown (defined by $K n_{\mathrm{GLL}-\max }>0.05$ ) occurs over a far smaller portion of the flowfield than for the $K n=0.3$ case. For the present case, continuum breakdown is limited to a region surrounding the bow shock, the forebody boundary layer, and a large portion of the wake. Figure 8 shows the boundaries between NS and BGK domains, at steady state, in a hybrid BGK-NS simulation of the $K n=0.03$ case. These boundaries, along which the right side of

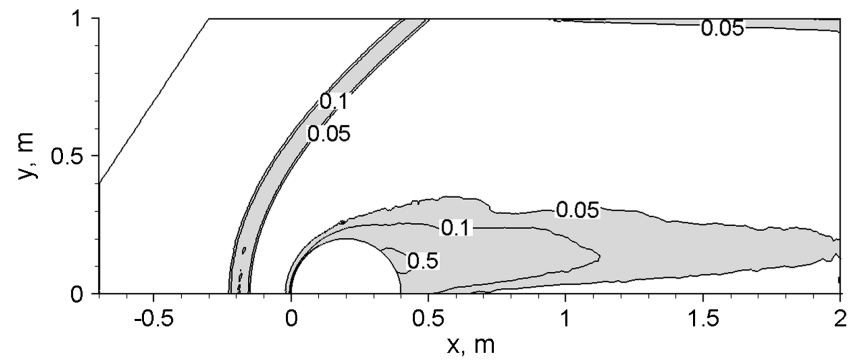

Fig. 7 Contours of the maximum-gradient-length local Knudsen number from the DSMC simulation at $K \boldsymbol{n}=\mathbf{0 . 0 3}$. Shaded area indicates regions of continuum breakdown.
Eq. (2) is equal to the cutoff value of 0.1 , correspond reasonably well to continuum breakdown boundaries shown in Fig. 7. Significant differences between domain boundaries in Fig. $\underline{8}$ and the 0.05 contour line in Fig. 7 are found only in the wake region.

In Fig. 9a, contours of normalized bulk velocity magnitude are shown for several different simulations at $K n=0.03$. Results are displayed for BGK, hybrid BGK-NS, hybrid BGK-Euler, DSMC, UFS NS and LeMANS NS simulations. Contour lines from both UFS NS and LeMANS NS simulations agree well with those from DSMC through much of the forebody shock layer, but noticeable disagreement is found in the wake. The BGK-Euler simulation gives particularly inaccurate results throughout the flowfield, and reduced accuracy is also found for the BGK simulation in wake regions far from the axis. Discrepancies observed between BGK and BGK-NS results in the wake seem to indicate that, in the hybrid BGK-NS simulation, the NS domain may include areas of strong nonequilibrium within the wake where BGK calculations would be more appropriate. This assumption is reinforced by differences, discussed above, between Figs. 7 and 8 . As expected, very good agreement is found between BGK, BGK-NS and DSMC simulations within the shock layer upstream of the cylinder.

Figures $\underline{9 b}$ and $9 \mathrm{c}$ show contours of normalized density and temperature, respectively, from simulations of the $K n=0.03$ case. Trends in both figures are similar to those in Fig. 9a. As in Fig. 9a, relatively good agreement is shown in Figs. $9 \mathrm{~b}$ and $\overline{\mathrm{g}} \mathrm{c}$ between most simulation results around the shock layer and forebody boundary layer, whereas particularly large discrepancies with DSMC are observed in results from the hybrid BGK-Euler simulation. The large error in BGK-Euler results is not particularly surprising, because (unlike BGK, DSMC, Boltzmann and NS calculations) Euler calculations completely neglect effects of diffusive transport and viscous dissipation, and any differences from a truly inviscid flow are due only to artificial viscosity.

In Fig. 10a, the variation in normalized velocity along the stagnation streamline is plotted for simulations of the $K n=0.03$ case. Very good agreement is found here between results from DSMC and LeMANS NS simulations. Excellent agreement is also shown in Fig. 10a between BGK and hybrid BGK-NS simulation results, although both give relatively poor agreement with DSMC toward the upstream portion of the shock. A comparison of BGK and DSMC results in this region indicates that the BGK approximation of the Boltzmann equation may produce excessive diffusive transport within the shock. As mentioned above in the discussion of Figs. 4, the same trend of excess diffusion in the shock is also found in a comparison of BGK, Boltzmann and DSMC results for the $K n=0.3$ case. In Fig. 10a, as in Figs. 9, the greatest differences from DSMC are observed in results from the hybrid BGK-Euler simulation. In relation to DSMC, the BGK-Euler simulation overestimates the shock standoff distance by approximately $12 \%$. Despite significant discrepancies in the location and thickness of the shock, all curves shown in Fig. 10a give relatively good agreement within the postshock and boundary-layer regions.

Figures $10 \mathrm{~b}$ and $10 \mathrm{c}$ show contours of normalized density and temperature, respectively, for the $K n=0.03$ case. Trends in both figures are similar to those in Fig. 10a, with comparable differences in shock thickness and shock standoff distance based on either density or temperature. The only prominent trends in Figs. 10b and

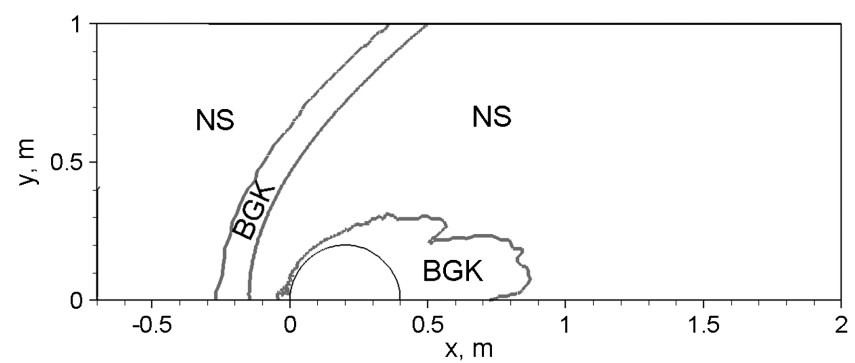

Fig. 8 Continuum and rarefied domains for the UFS BGK-NS simulation at $K n=0.03$. 

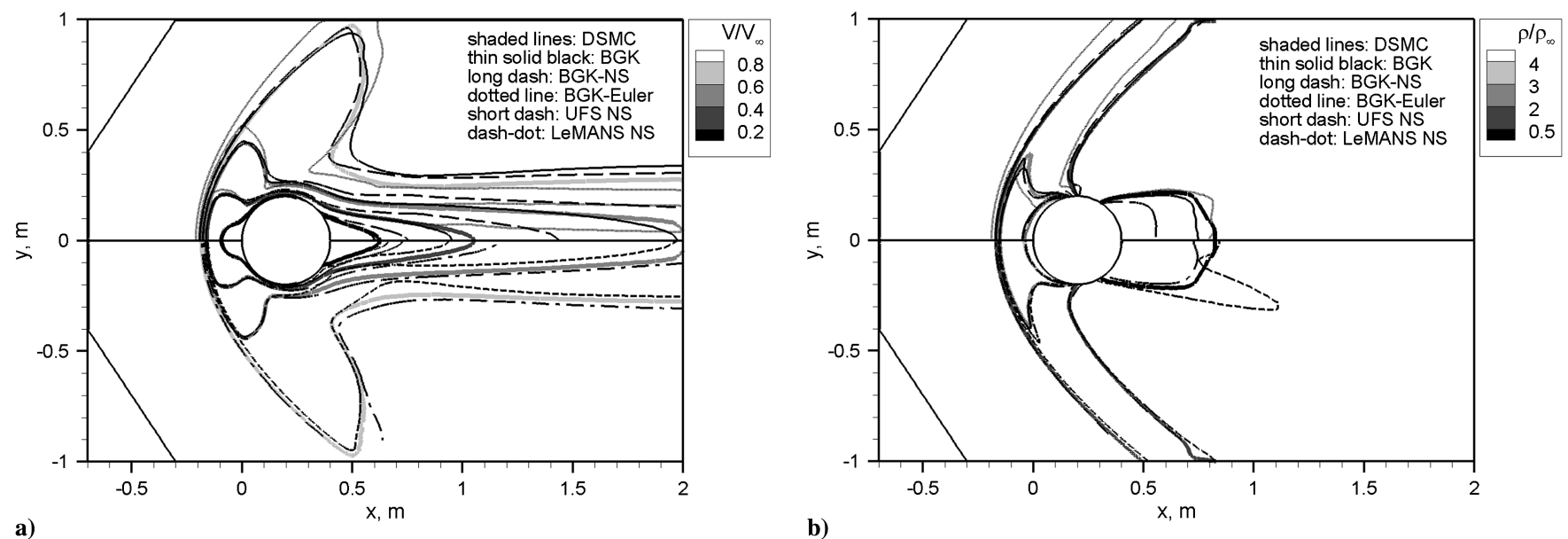

a)

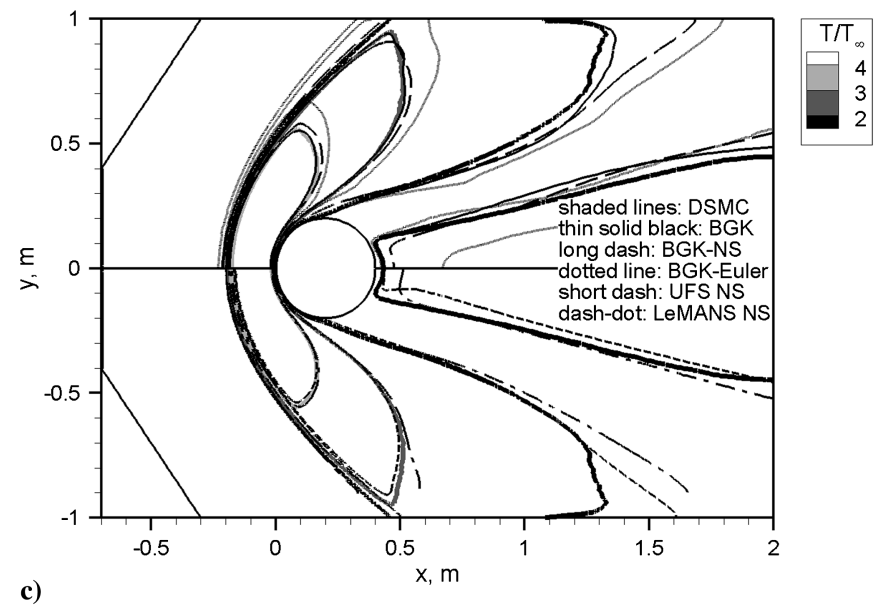

Fig. 9 Field property contours for $K n=0.03$ : a) bulk velocity magnitude, b) density, and c) temperature. UFS is used for all simulations except the LeMANS NS simulation and the MONACO DSMC simulation.

10c that are not apparent in Fig. 10a relate to boundary-layer thickness. The BGK, BGK-NS and BGK-Euler simulations all noticeably overestimate the boundary-layer thickness, as measured by density or temperature, relative to DSMC. Corresponding gradients within the boundary layer are lower in these simulations than in DSMC. In contrast, only very small differences in boundarylayer thickness are found in Figs. 10b and 10c between results from LeMANS NS and DSMC simulations. Differences in boundarylayer thickness are likely due to excess diffusive transport in BGK calculations; this may be attributed to inaccuracy in the BGK approximation of the collision term in the Boltzmann equation and is also thought to cause the overly thick shocks observed in Figs. 4 and 10a.

Surface pressure coefficients for simulations at $K n=0.03$ is plotted in Fig. 11a. Good agreement is observed along the forebody between all pressure coefficient curves, whereas significant quantitative (but not qualitative) differences are found for $\theta>90^{\circ}$. The greatest disagreement with DSMC pressure coefficient values at $\theta>90^{\circ}$ is shown in results from the BGK-Euler and NS simulations. As discussed above, error in NS results is likely due to effects of continuum breakdown, whereas BGK-Euler inaccuracies can be mainly attributed to a lack of diffusive transport in calculations for the Euler equations. Although BGK-NS and NS results are in good agreement for $\theta>150^{\circ}$, neither compares well with BGK or DSMC results over this portion of the surface. This trend seems to indicate that, as mentioned in the discussion of Fig. 9a, the NS domain in the BGK-NS simulation may include rarefied wake regions that should be assigned to the BGK domain.

Figure $11 \mathrm{~b}$ shows the variation in the surface friction coefficient for simulations of the $K n=0.03$ case. The most noticeable errors are found in the NS curve, which agrees reasonably well with DSMC over the forebody surface $\left(\theta<90^{\circ}\right)$ but is up to a factor of 5 greater than DSMC along the afterbody. Most of this discrepancy can be attributed to the lack of a velocity slip/temperature jump boundary condition in NS calculations; due to the high degree of continuum breakdown along the cylinder surface shown in Fig. 7, significant velocity slip is expected. Both DSMC and BGK calculations allow for velocity slip along the surface, and as expected, the lack of wall slip in NS calculations is associated with higher friction coefficient values in the NS results. All UFS simulations tend to slightly overpredict surface shear stress along the forebody, with an overestimate relative to DSMC of up to around $50 \%$ in the BGK results. Note the slope discontinuity in all curves around $\theta=165^{\circ}$, which corresponds to boundary-layer separation at the edge of a recirculation zone. In comparing the location of this discontinuity between results from different simulations, we find good overall agreement in the size of the recirculating region. Relative to DSMC, the maximum difference in the $\theta$ range for the recirculating region is found in results from the BGK-NS simulation, which overestimates this range by about $30 \%$.

In Fig. 11c, the surface heat transfer coefficient is plotted for simulations at $K n=0.03$. As with the friction coefficient values shown in Fig. 11b, the NS simulation slightly overpredicts the heat transfer over much of the forebody surface and greatly overpredicts this quantity along the afterbody. A maximum relative error (with respect to the DSMC value) of approximately $32 \%$ is found at $\theta=148^{\circ}$. Significant discrepancies are observed between DSMC and the various UFS results, with a roughly $20 \%$ underestimate in heat transfer over much of the surface from the BGK-NS simulation and a somewhat smaller overestimate from the BGK simulation.

The underestimate in BGK-NS heat flux values in Fig. 11c is likely due primarily to some combination of the following factors: First, an overly small temperature gradient (relative to DSMC) is 


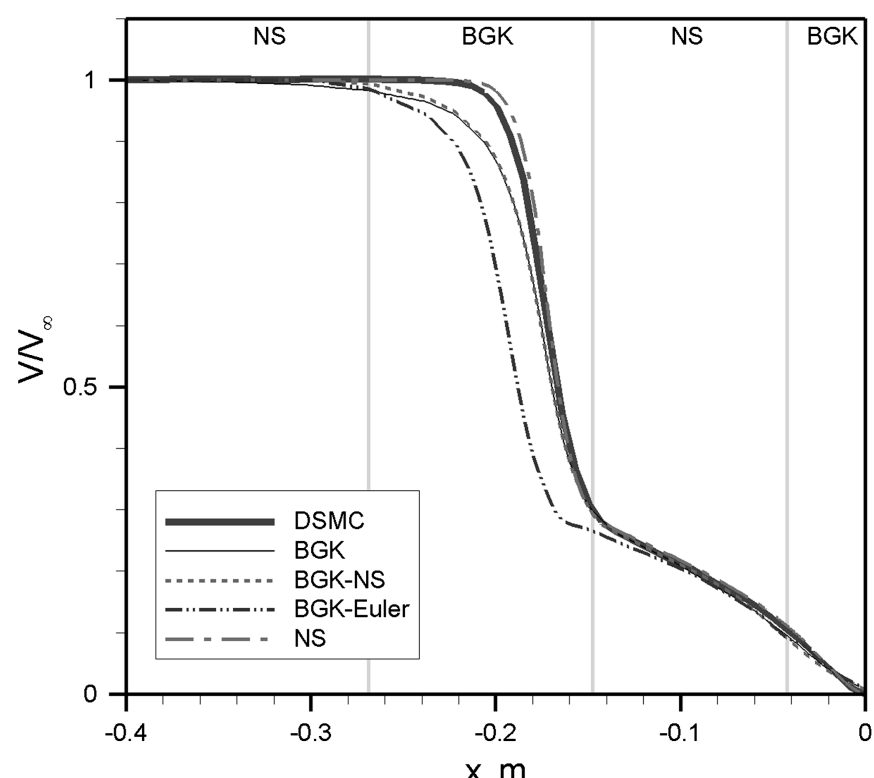

a)

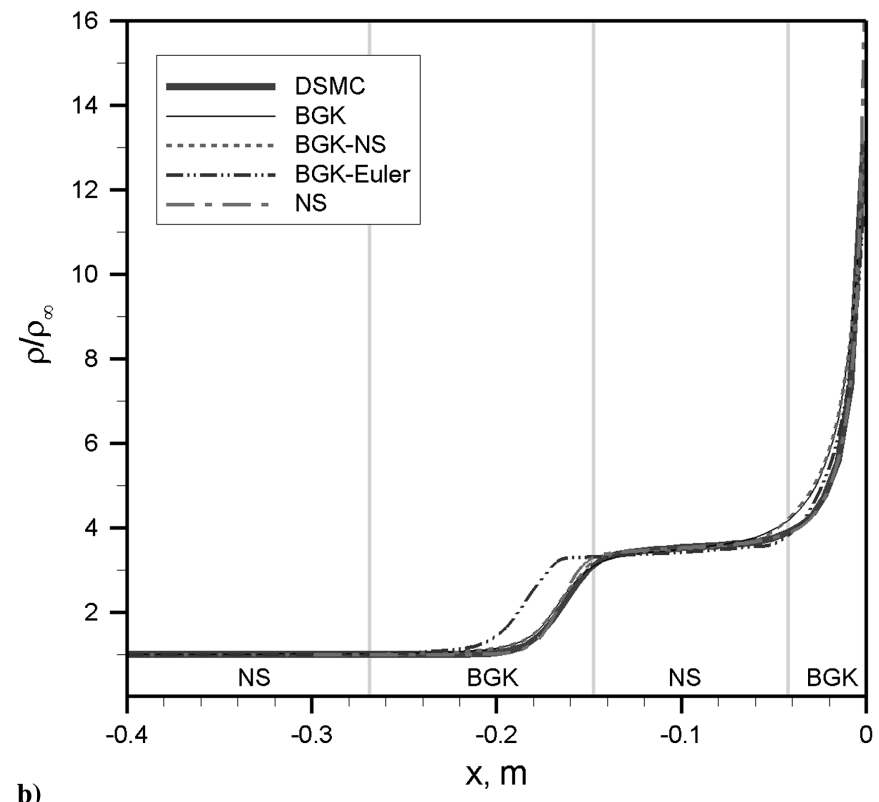

b)

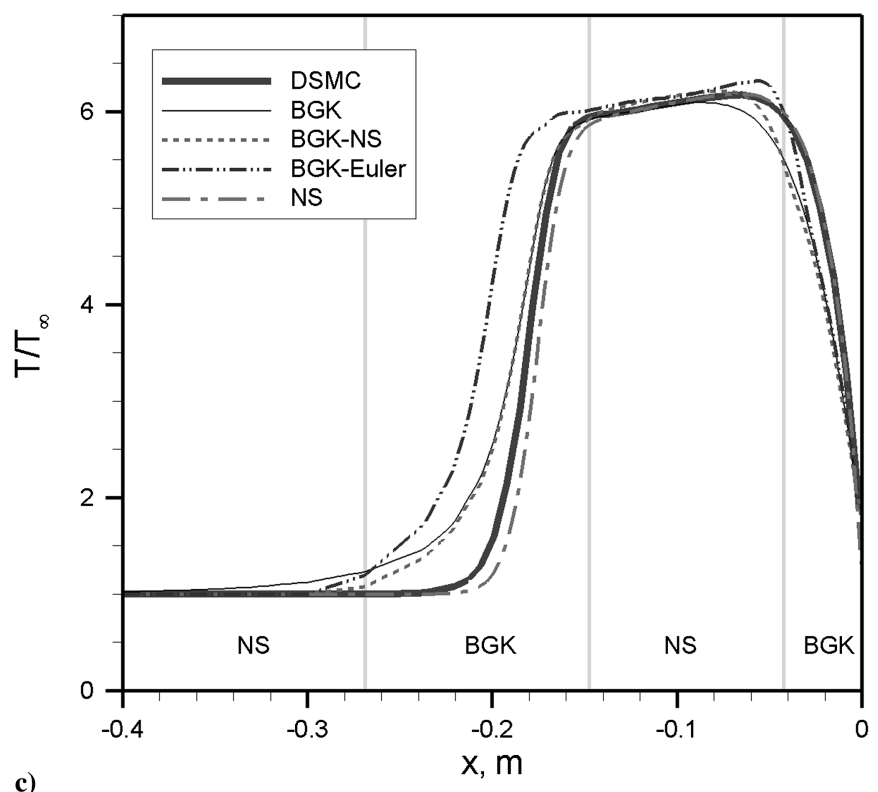

Fig. 10 Property variation along the stagnation streamline for $K n=0.03:$ : a) bulk velocity magnitude, b) density, and c) temperature. UFS is used for BGK, BGK-NS, and BGK-Euler simulations.

present through much of the forebody boundary layer, as shown in Fig. 10c. Second, the lack of a Prandtl number correction in BGK calculations, as mentioned above in the discussion of Fig. $5 \mathrm{c}$, results in an unphysically low thermal conductivity, which should further reduce surface heat flux. Finally, linearization of collision term in the BGK equation should reduce accuracy in surface heat flux and may contribute to errors in NS-BGK simulation results shown in Fig. 11c. Note that, in contrast to the NS-BGK results in this figure, an overestimate in heat flux is observed over much of the forebody surface for the BGK simulation. Although no clear explanation for this trend is apparent, possible contributors include unphysically high numerical diffusion due to approximations in the BGK collision operator.

As mentioned in the discussion of Figs. 5, UFS simulation results in Figs. 11 exhibit an unphysical stair-step pattern in the $\theta$ range between $\overline{90}$ and $150^{\circ}$. These errors, as well as a lack of smoothness in surface heat transfer curves at smaller $\theta$ values, are likely caused by problems with gradient evaluations involving Cartesian cut cells. Note that UFS BGK and NS calculations are entirely deterministic, and fluctuations observed in the UFS results are not due to statistical scatter as found in results from DSMC simulations.

\section{Comparison of Simulation Results for $\boldsymbol{K} \boldsymbol{n}=\mathbf{0 . 0 0 3}$}

Figure 12 shows the computational meshes used in UFS NS and LeMANS $\bar{N} S$ simulations at $K n=0.003$. Although the UFS mesh is able to capture high gradient regions within areas of increased refinement, no automatic grid adaption is possible using LeMANS. The LeMANS mesh, which was manually generated using a commercial code, includes a structured region surrounding the cylinder and an unstructured region of uniform-sized triangular cells that covers much of the wake. In creating the LeMANS mesh, particular effort was made to ensure shock alignment and grid independence. As described in section III, stretched (i.e., high aspect ratio) cells are used in the boundary layer to avoid unnecessarily small cell dimensions in the surface-tangent direction.

In Fig. 13, contours are shown for the maximum-gradient-length local Knudsen number, as computed from the LeMANS simulation at $K n=0.003$. As expected for such a small Knudsen number value, continuum breakdown (where $K n_{\mathrm{GLL}-\max }>0.05$ ) is found to occur only in narrow regions around the bow shock and forebody boundary layer, as well as in portions of the near-field wake region. This indicates that the NS equations are valid over nearly the entire 


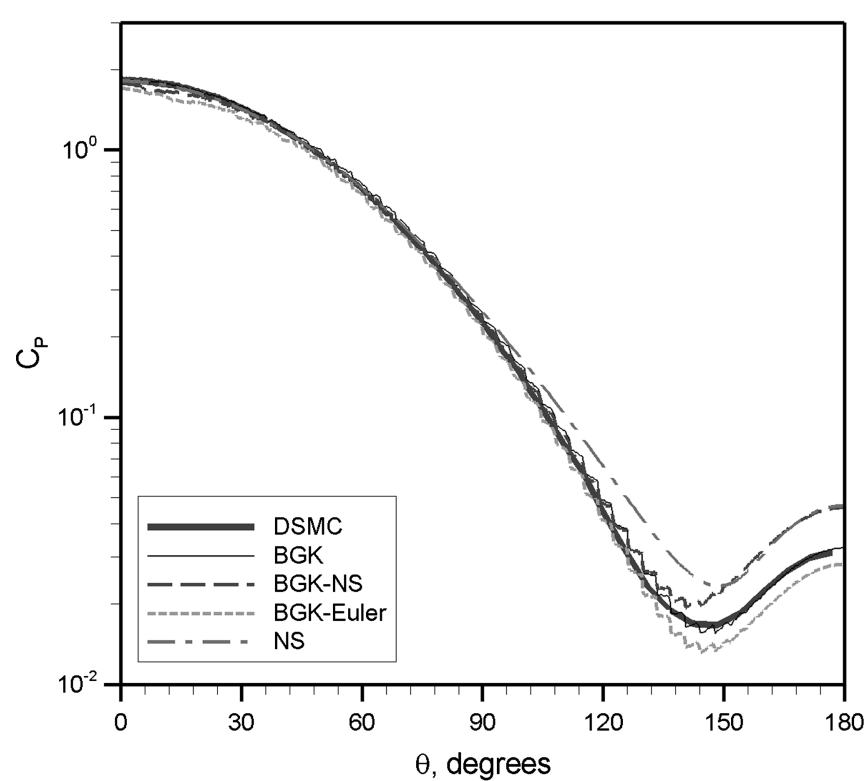

a)

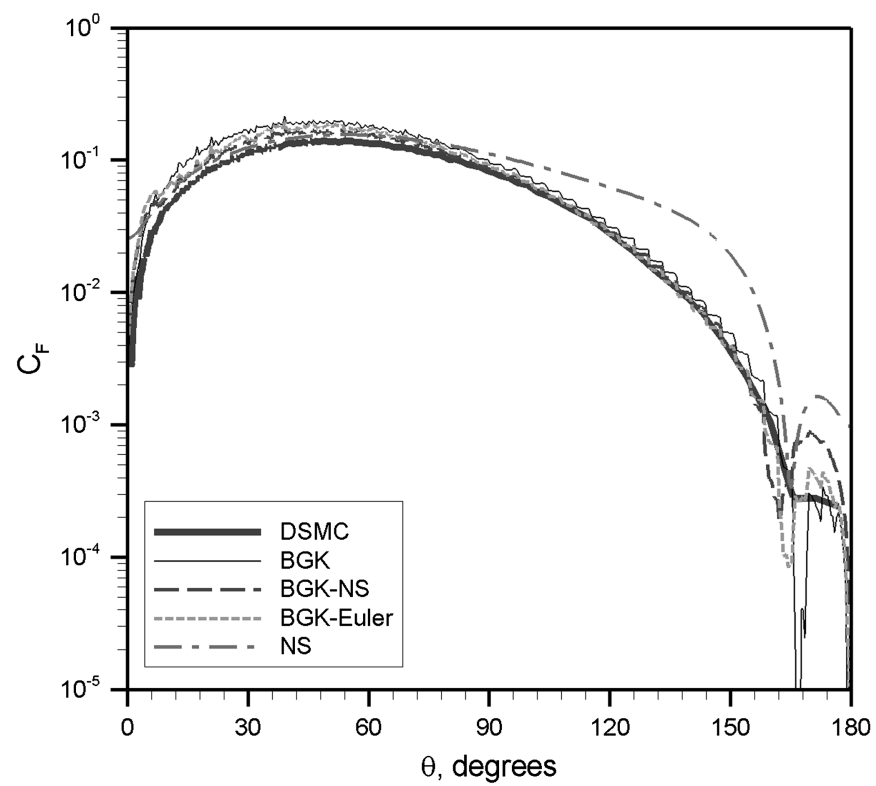

b)

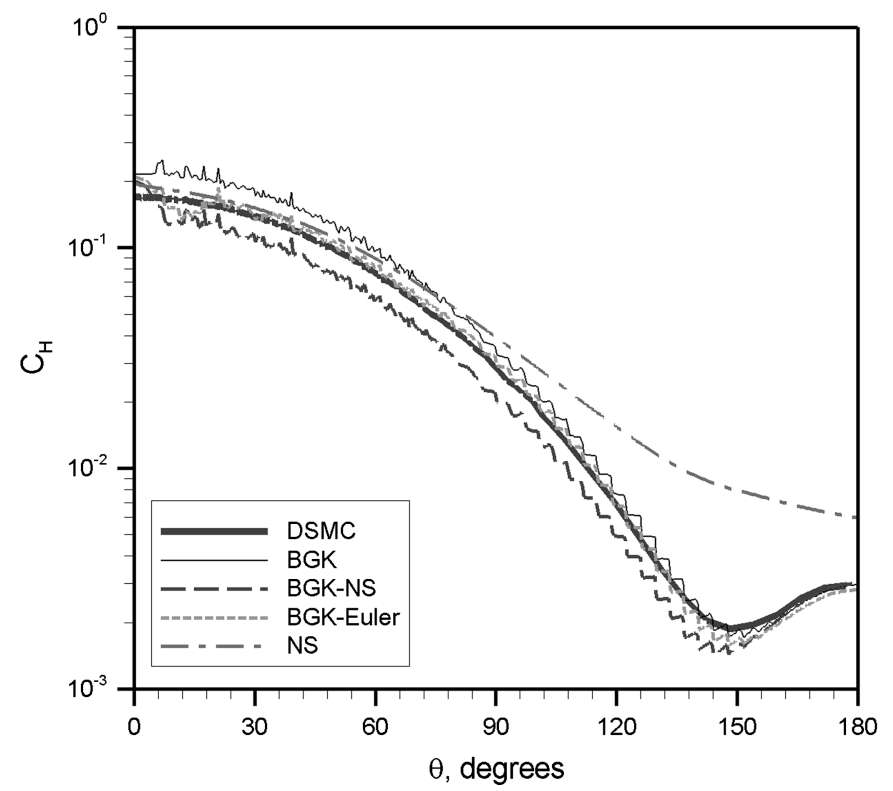

c)

Fig. 11 Surface flux coefficients for $K n=0.03$ : a) pressure, b) friction, and c) heat transfer. UFS is used for BGK, BGK-NS, and BGK-Euler simulations.

flowfield, and relatively small errors should result from the nearequilibrium assumptions underlying these equations.

In Fig. 14a, contours of normalized bulk velocity magnitude are compared from the two NS simulations at $K n=0.003$. Very good overall agreement is found between the two sets of contour lines, with the largest discrepancies observed in the far-field wake region. Differences in the shape of the $V / V_{\infty}=0.8$ contour line far from the axis may be attributed to the presence of a UFS symmetry boundary condition at $y=1 \mathrm{~m}$. Particularly good agreement is found in the shock layer, with a shock standoff distance based on velocity contours that is approximately 5\% larger in the LeMANS simulation than in the UFS simulation. Figures $14 \mathrm{~b}$ and $14 \mathrm{c}$ show contours of normalized density and temperature, respectively, from the $K n=$ 0.003 simulations. In both figures, levels of disagreement are similar to those in Fig. 9c, with the largest discrepancies in the postshock region far from the axis and in the wake.

The variation in bulk velocity, density and temperature along the stagnation streamline is plotted in Figs. 15 for the NS simulations at $K n=0.003$. All three figures show very similar trends, with excellent agreement in postshock values and boundary-layer profiles. However, the LeMANS simulation gives a noticeably thicker shock and a slightly larger shock standoff distance. Based on the location of maximum gradients, the UFS simulation is found to underestimate the shock standoff distance by between $4 \%$ and $5 \%$ relative to the LeMANS simulation.

The reasonably good overall agreement shown in Figs. 14 and 15 can be seen to indicate that the Xu scheme [18] implementation in

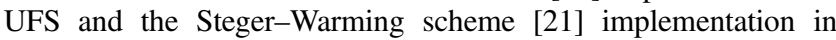
LeMANS both accurately represent the underlying physics of the NS equations, and both provide accurate results over the Knudsen number regimes for which the NS equations are valid. Much of the disagreement observed between LeMANS and UFS NS results in Figs. 14 and 15 can be attributed to the use of different assumptions and approximations between Xu [18] and Steger-Warming [21] schemes. In particular, disagreement in shock thickness may be due to differences in shock-capturing resolution, which are in turn caused by greater numerical diffusion within high pressure gradient regions in the Steger-Warming NS calculations. 


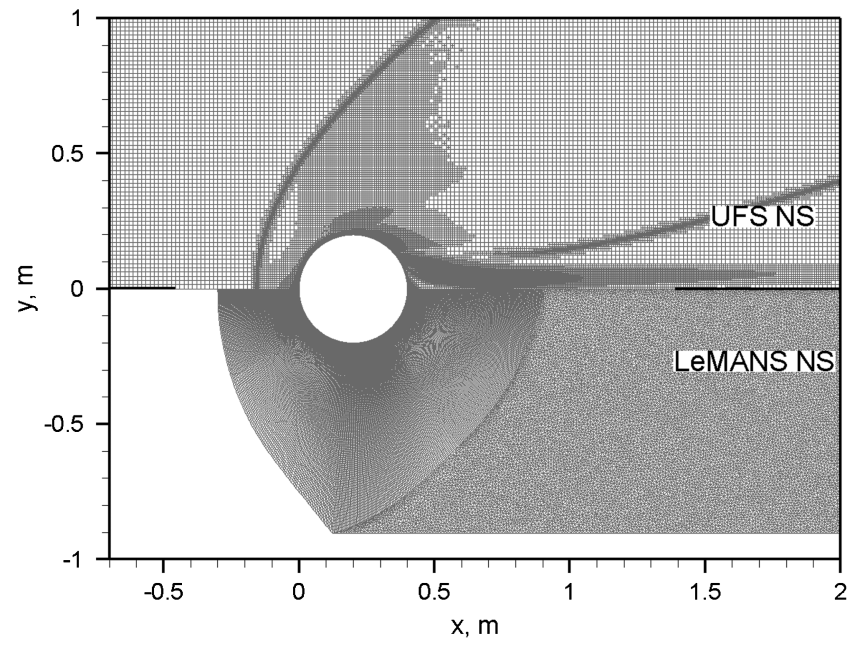

Fig. 12 Meshes for UFS NS and LeMANS NS simulations at $K n=0.003$.

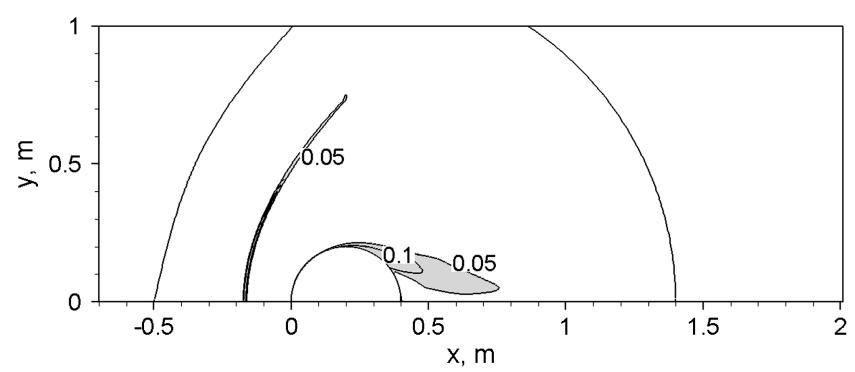

Fig. 13 Contours of the maximum-gradient-length local Knudsen number from the LeMANS simulation at $K n=0.003$. Shaded area indicates regions of continuum breakdown.

\section{Drag Coefficient Comparison}

In Fig. 16, drag coefficients $C_{d}$ calculated from Boltzmann, BGK, DSMC, and NS simulations are plotted as a function of Knudsen number. Data from experiments of Maslach and Schaaf [24] are also included in the figure for comparison. Figure 17 includes data points from a number of additional simulations and is otherwise identical to Fig. 16. Symbols in Fig. 17 labeled "Tratio $=1.2$ " indicate that the ratio of cylinder wall temperature to freestream temperature is 1.2 , as is the case for all simulations described above and included in Fig. 1. Likewise, symbols labeled "Tratio $=4$ " indicate simulations for which the cylinder wall temperature is four times that of the freestream gas. Several additional simulations using this higher wall temperature have been performed over a wide range of Knudsen number values. Among these added simulations, five are run using the LeMANS NS solver, seven use MONACO, and seven employ the BGK module in UFS. Additional data points, labeled "Boltzmann* Tratio $=4 "$ are taken from published results of UFS Boltzmann simulations by Kolobov et al. [12].

In Fig. 17, the cylinder surface temperature is shown to have little if any noticeable impact on $C_{d}$ over the full Knudsen number range considered. Generally good agreement is found between UFS Boltzmann, DSMC and measured values even around the maximum Knudsen number of 10 . Good agreement is also found between all UFS, DSMC, NS and experimental data for $K n<0.1$. At higher Knudsen numbers, however, large overestimates relative to the experimental values are observed in NS $C_{d}$ predictions, with an overestimate of roughly $70 \%$ at $K n=1$. Smaller but still significant errors in $C_{d}$ are shown in BGK results for $K n>0.3$, with an underestimate relative to DSMC of about $7 \%$ at $K n=10$. A noticeable increase with Knudsen-number is found in the level of disagreement between DSMC and BGK results. As no such Knudsen-number-dependent discrepancies are found between DSMC and Boltzmann results, this disagreement may be attributed

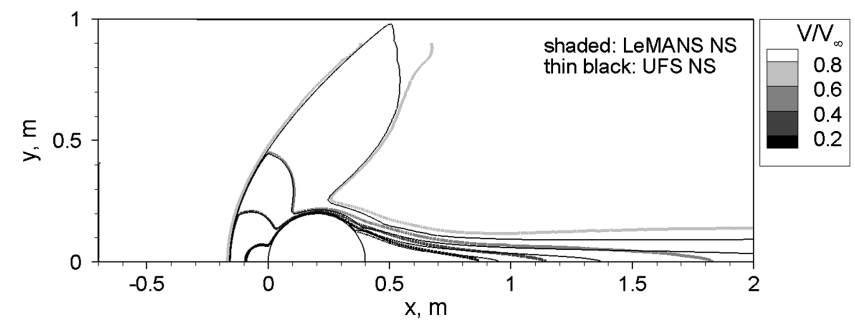

a)

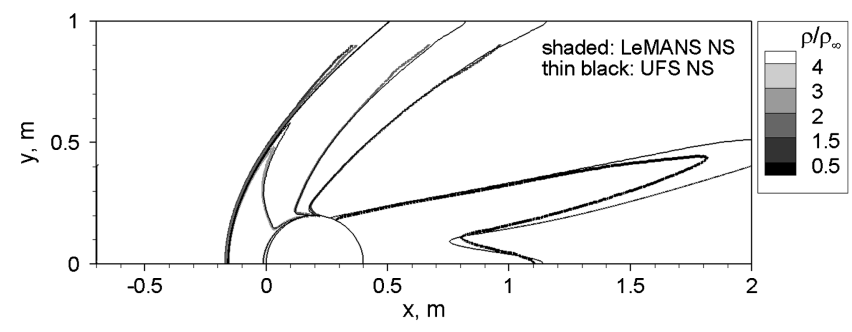

b)

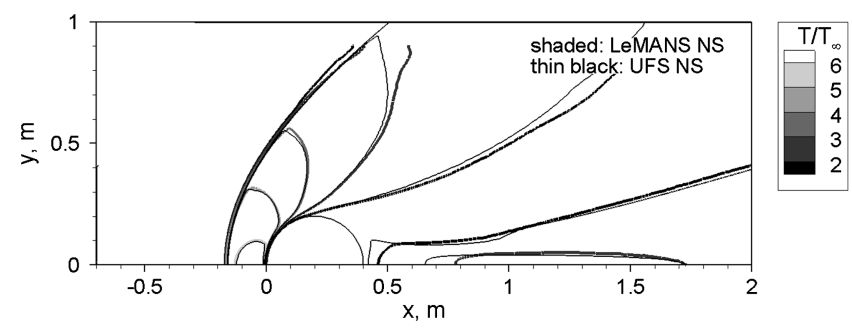

c)

Fig. 14 Field property contours for $K n=0.003$ : a) bulk velocity magnitude, b) density, and c) temperature.

to inaccuracies in the BGK collision integral approximation that become increasingly influential at higher Knudsen numbers.

\section{Relation of Observed Trends to Discrepancies in Expansion Flow Results}

In a recent study of Josyula et al. [14], a rarefied nozzle/plume expansion flow is simulated using both $\overline{\mathrm{DSMC}}$ and the hybrid BGKEuler option in UFS, and significant differences are observed between the two sets of results. In particular, the centerline density in the plume is overestimated by up to $28 \%$ in the UFS simulation. Significant differences are also found in the thickness, position and maximum temperature of the mixing layer, which separates ambient and plume flow regions. Relative to DSMC, the hybrid BGK-Euler simulation is shown to overestimate the mixing-layer thickness based on either density, translational temperature or Mach number contours.

Figure 18, reproduced with permission from [14], shows Mach number contours within the plume from UFS and DSMC simulations. Here, vibrationally excited nitrogen is ejected from a diverging nozzle, with a throat diameter of $0.16 \mathrm{~cm}$ and an area ratio of 8 , into a low-density ambient background. Among the differences found in a comparison of the upper (UFS) and lower (DSMC) portions of the figure, the UFS simulation gives a significantly thicker mixing layer, higher Mach numbers along both symmetry axes, and reduced Mach numbers along the inner edge of the mixing layer.

Figure 19 shows profiles of translational temperature along a radial plane located $1.15 \mathrm{~cm}$ downstream of the nozzle exit. Vertical lines in Fig. 19 denote boundaries between BGK and Euler domains in the UFS simulation. As shown in the figure, the centerline temperature is around $10 \%$ higher in the UFS BGK-Euler simulation, the location of maximum translational temperature is slightly further from the centerline in the UFS simulation results, and a wide mixing-layer "tail" projecting radially outward in the DSMC translational 

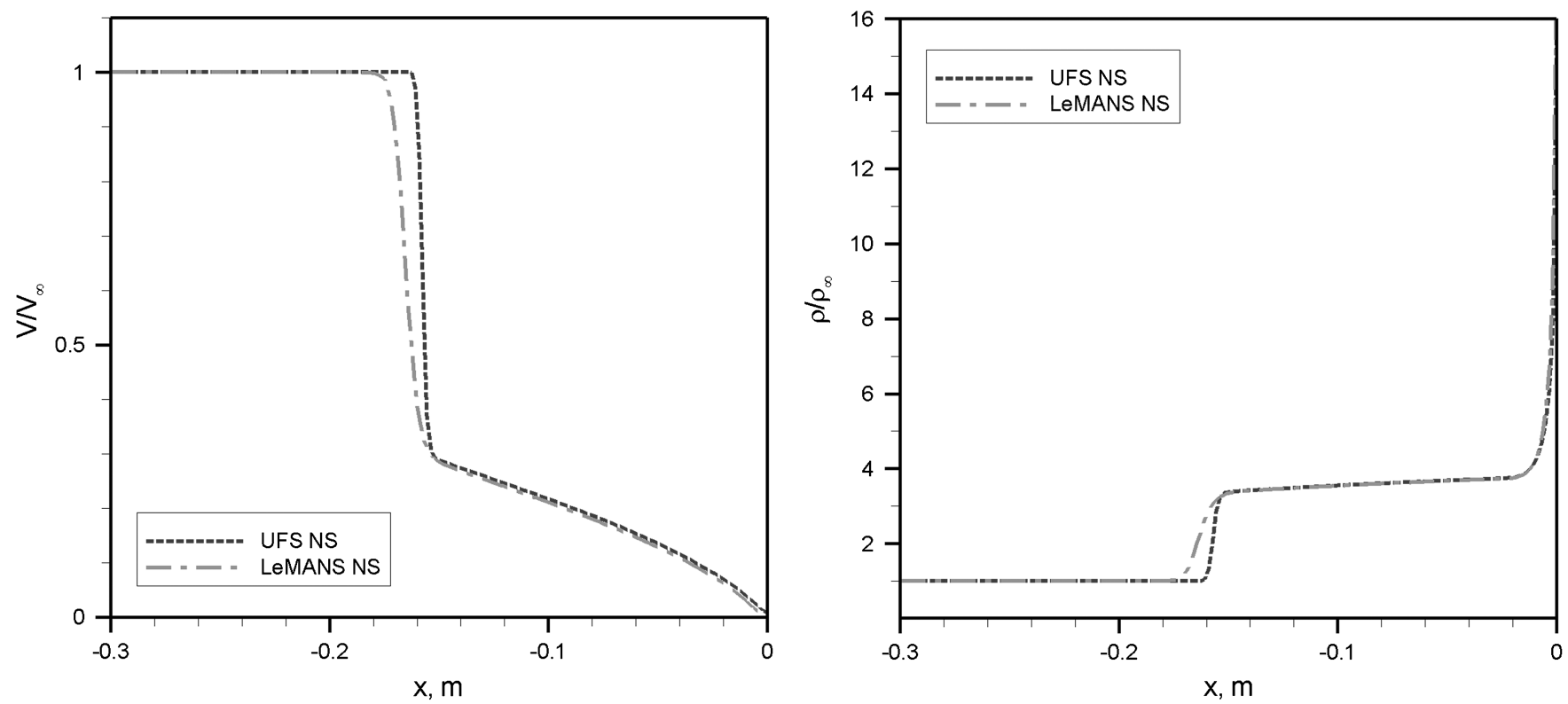

a)

b)

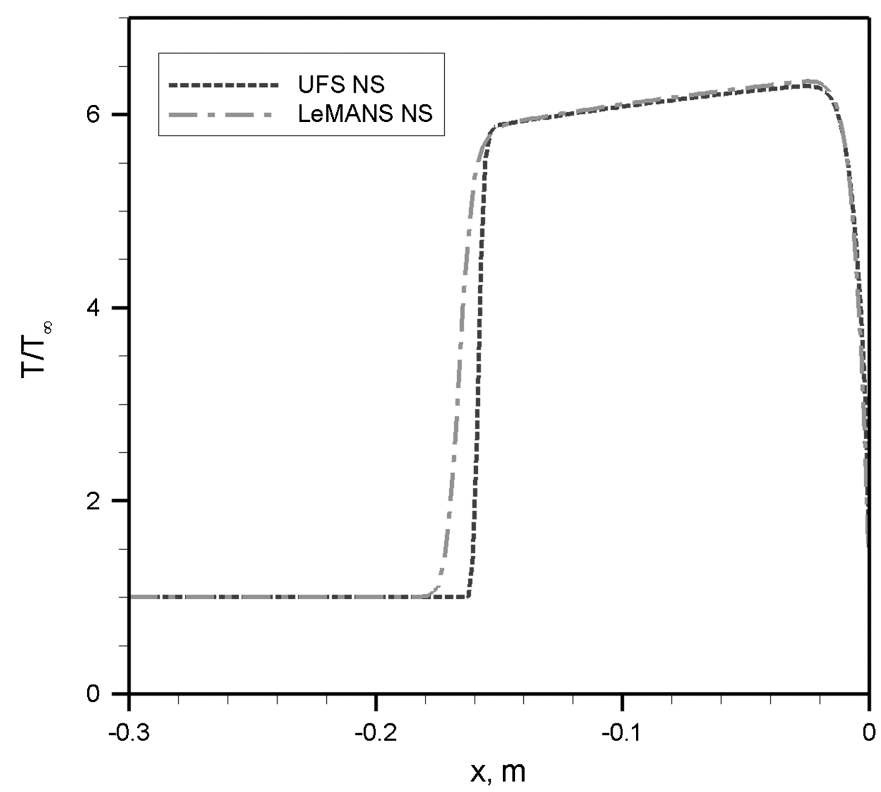

c)

Fig. 15 Property variation along the stagnation streamline for $K n=0.003$ : a) bulk velocity magnitude, b) density, and c) temperature.

temperature profile is absent in the corresponding profile from the UFS simulation. Although several of these differences are not clearly explained in [14], trends observed in the present study can provide some insight on the causes of the discrepancies.

First, the overestimate of mixing-layer thickness in UFS results of Josyula et al. [14] can be partly explained by numerical diffusion effects in the UFS BGK solver, which are shown to cause an unphysically large shock thickness in Figs. 4 and 10. As found in both the present study and [14], increased numerical diffusion in BGK calculations, relative to full Boltzmann or DSMC calculations, seems to be independent of velocity or spatial grid resolution. Based on comparisons between BGK and Boltzmann results in Figs. 4 and 10 , the problem may therefore be attributed to inherent inaccuracy of the BGK equation within high Knudsen number regimes.

The same hybrid BGK-Euler approach used in [14] is also employed here for the $K n=0.03$ case, although unlike [14], the current study focuses on a monatomic gas for which no consideration of internal energy relaxation is required. Figures 10a-10c show increased shock standoff distance for the BGK-Euler simulation relative to all other simulations performed for the same flow.
Although the cause of this error is not entirely clear, it may be due to a lack of consideration for physical diffusive transport within the continuum domain, as discussed above. The overestimate in the mixing-layer radial position in BGK-Euler results from [14] may be seen as a qualitatively similar error and is therefore likely attributable to the same cause.

A wide region of elevated temperature is located radially outward from the mixing layer in DSMC results of Josyula et al. [14], but is not present in corresponding BGK-Euler results. [See Figs. 21(b) and 23(b) in [14].] This discrepancy is explained in [14] as an error in DSMC calculations associated with the presence of a nearby freestream inflow boundary. Although the explanation in [14] cannot be discounted, another likely contributor to this discrepancy is the fact that the elevated temperatures are found in a region of very low collision frequency within the Euler domain in the BGK-Euler simulation. As the Euler equations assume an infinitely high collision frequency, any radially outward diffusion of thermal energy from the mixing layer is suppressed in BGK-Euler calculations, and the long mixing-layer tail shown in the DSMC temperature profile should be terminated along the boundary between BGK and Euler domains. 


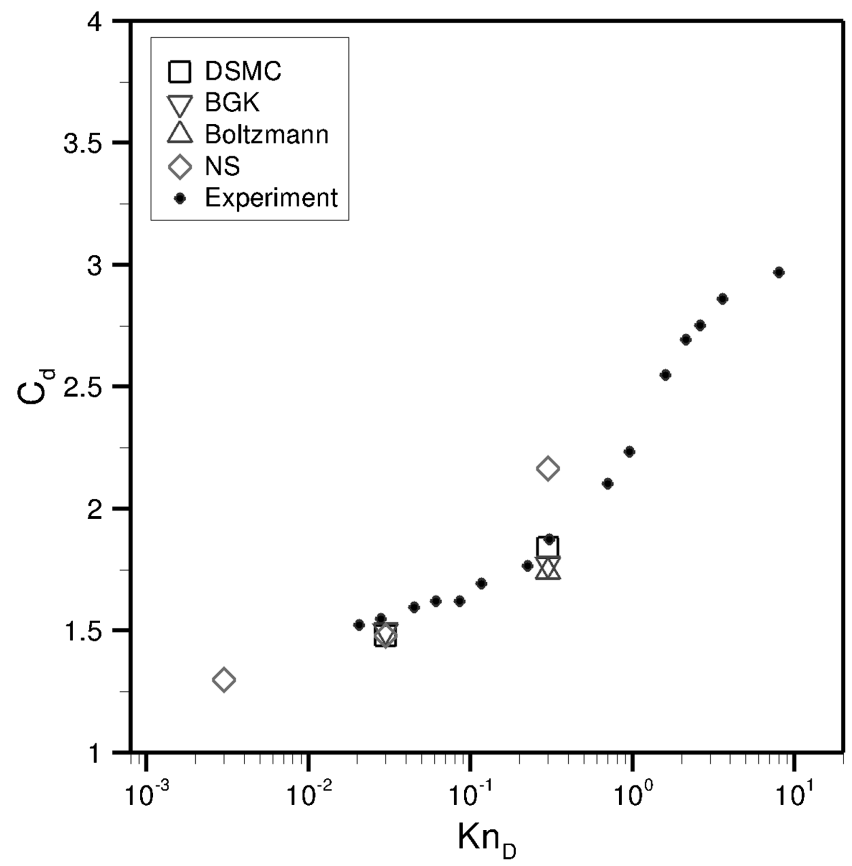

Fig. 16 Variation in drag coefficient with Knudsen number.

A qualitatively similar effect is shown in Fig. 10c, where a sharp cutoff in the temperature boundary layer is observed along the continuum breakdown boundary in BGK-Euler simulation results for the $K n=0.03$ case.

\section{Efficiency Comparison}

Table 1 shows a summary of numerical parameters for the 12 different UFS, DSMC, and LeMANS simulations that are used to generate the results shown in Figs. 2-15. One important feature in the table is the enormous difference in expense between DSMC and Boltzmann simulations at $K n=0.3$. The DSMC simulation for this case requires less than $0.2 \%$ of the CPU time needed for the Boltzmann simulation, which corresponds to a difference of over two orders of magnitude in computational expense. Although poor Boltzmann solver efficiency relative to DSMC is expected for a

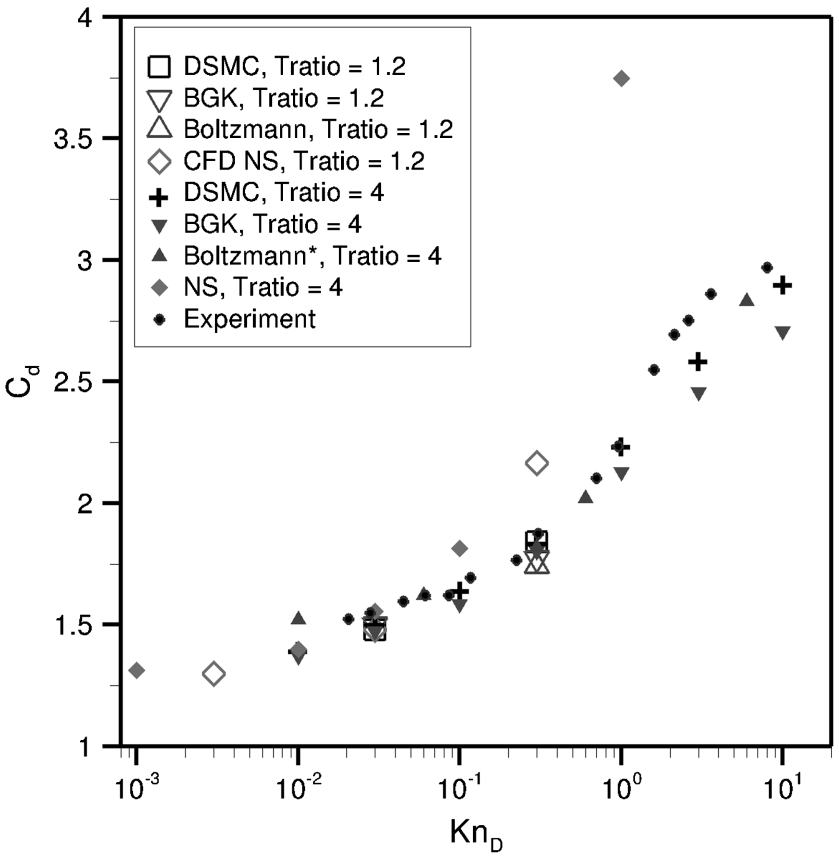

Fig. 17 Variation in drag coefficient with Knudsen number, with results from additional simulations.

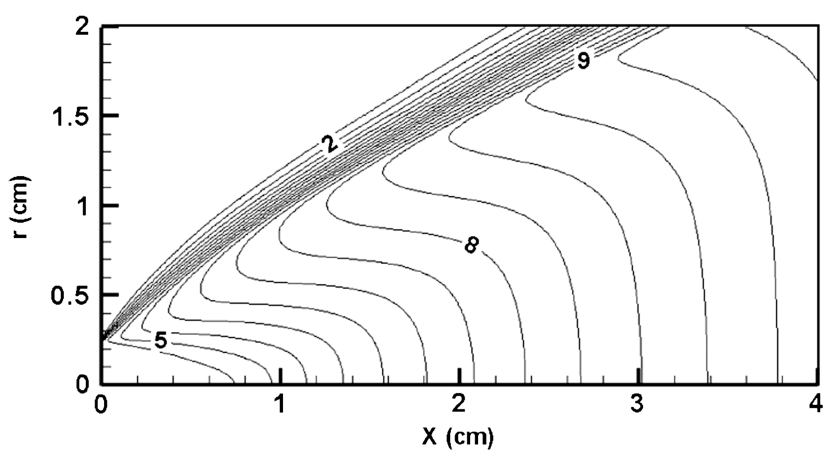

a)

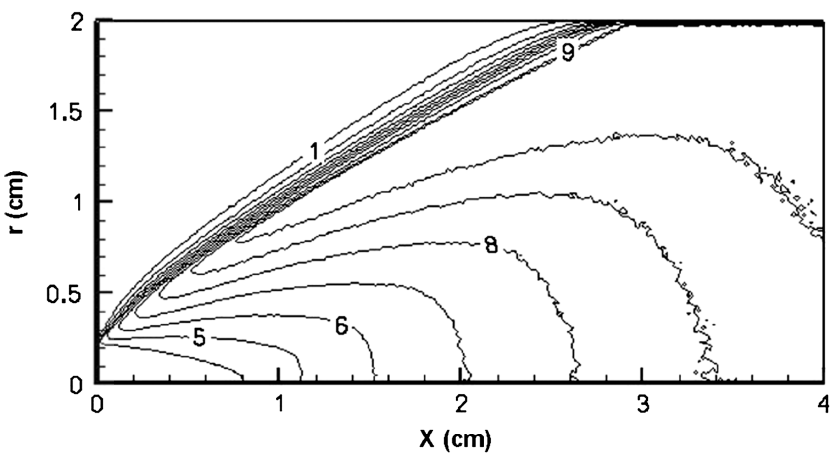

b)

Fig. 18 Plume Mach number contours for UFS BGK-Euler (top) and DSMC (bottom) simulations from [14]. Reproduced with permission.

supersonic flow problem, the magnitude of the difference between DSMC and Boltzmann CPU times is surprising. However, we expect that careful adjustment of the Boltzmann velocity grid domain and refinement level could considerably reduce CPU time for the Boltzmann simulation. Such adjustments would involve balancing various tradeoffs between accuracy and efficiency, which is an active research area [25] but is outside the scope of the present work. As there is currently no systematic technique for velocity grid sizing in a Boltzmann calculation, we have used a velocity grid spacing and domain size conservatively based on standard guidelines as given in UFS documentation.

The very large relative expense of the Boltzmann simulation can be primarily attributed to a few main factors, in addition to the use of potentially nonoptimal velocity grid parameters: First, Boltzmann calculations are performed on a uniform velocity space grid that is not adapted for local efficiency optimization. A relatively large number of nodes are therefore required to adequately approximate

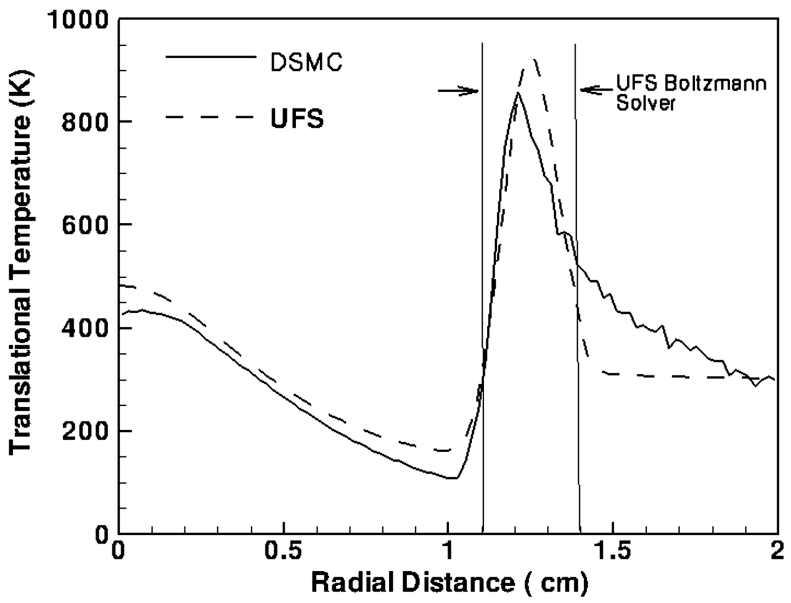

Fig. 19 Translational temperature profiles in the plume for UFS BGKEuler and DSMC simulations from [11]. Profiles are taken $1.15 \mathrm{~cm}$ downstream of the nozzle exit plane. Reproduced with permission. 
Table 1 Comparison of simulation parameters and CPU time for UFS, MONACO, and LeMANS simulations

\begin{tabular}{lccccc}
\hline \hline Simulation method & $K n$ & Cells & Velocity space nodes & Particles & CPU h \\
\hline Boltzmann (UFS) & 0.3 & 6,515 & 32,000 & - & 1929.4 \\
BGK (UFS) & 0.3 & 6,605 & 3,200 & - & 18.5 \\
NS (LeMANS) & 0.3 & 1,4261 & - & 4.47 \\
DSMC (MONACO) & 0.3 & 3,735 & - & 107000 & 3.75 \\
BGK (UFS) & 0.03 & 23,208 & 3,200 & - & 66.5 \\
BGK-NS (UFS) & 0.03 & $10,490(582$ NS) & 3,200 & - & 60.0 \\
BGK-Euler (UFS) & 0.03 & $11,271(874$ Euler) & 3,200 & - & - \\
NS (UFS) & 0.03 & 19,719 & - & - & - \\
NS (LeMANS) & 0.03 & 101,959 & - & $1,860,000$ & 64.3 \\
DSMC (MONACO) & 0.03 & 65,189 & - & - & 50.9 \\
NS (UFS) & 0.003 & 72,446 & - & - & 48.0 \\
NS (LeMANS) & 0.003 & 101,959 & - & - \\
\hline \hline
\end{tabular}

the shape of the velocity distribution within both high- and lowMach-number regions. In addition, and in contrast to BGK simulations, a three-dimensional velocity grid is required for simulations of a two-dimensional flow. This accounts for the fact that, as shown in the table, 10 times more velocity grid nodes are used in the Boltzmann simulation than in simulations employing the BGK module in UFS.

Another contributing factor for the high computational cost of the Boltzmann solver is increased sensitivity, relative to DSMC, of a discrete velocity Boltzmann simulation to the number of collision operations per time step. A large number of simulated collisions are typically required in Boltzmann calculations for sufficiently small statistical scatter in approximating the velocity distribution function [12]. Factors contributing to the large required number of collisions include a lack of time-averaging for simulation results, optional coupling with NS or Euler calculations in a hybrid simulation and, in particular, the relatively small contributions to the collisional relaxation process for any simulated collision that involves incident velocities in the low-probability tails of the velocity distribution.

As discussed above, numerical solutions to the Boltzmann equation on discrete velocity grids should have comparable accuracy with DSMC and hold potential advantages to DSMC in simulating a variety of rarefied or multiscale gas flows. Discrete velocity Boltzmann methods should be particularly promising for unsteady or subsonic flows, flows for which the tails of the velocity distribution must be characterized with high precision, or cases for which strong coupling with a NS solver is desired. Still, for this particular case it seems reasonable to conclude that DSMC simulation is preferable to simulation using the UFS Boltzmann solver, based on considerations of computational expense.

As shown in Table 1, the DSMC simulation for $K n=0.03$ is almost equally expensive as the corresponding BGK simulation, and BGK is roughly five times more expensive than DSMC at $K n=0.3$. The apparent increase in relative computational cost for BGK calculations at higher $K n$ can be attributed almost entirely to Knudsen number dependence in the ratio of cells between BGK and DSMC simulations. Cell size is adapted in BGK and DSMC calculations based, respectively, on flow property gradients and the local mean free path. This leads to somewhat different Knudsen number scaling in the number of cells for BGK and DSMC simulations.

As expected, efficiency gains are found in the hybrid BGK-NS and BGK-Euler simulations in comparison to the full BGK simulation at $K n=0.03$, although these gains are smaller than $15 \%$ for both hybrid simulations. Given the added complexity of hybrid scheme implementation and the fact that BGK and NS calculations should have comparable accuracy within continuum regions, the similar level of computational expense for BGK and hybrid UFS simulations at $K n=0.03$ seems to indicate that hybrid techniques are not preferable for this case.

Computational expense is comparable for UFS NS and LeMANS simulations at $K n=0.003$, with fewer cells but more iterations in the UFS simulation. Note that the difference in cell count between these two simulations is due in large part to the use of gradient-based mesh adaptation in UFS, which allows for larger cells in low-gradient regions. This difference is partially offset by the use of stretched (i.e., high aspect ratio) cells near the cylinder surface in the LeMANS simulation; UFS requires that square cells be used, so far more cells are needed near the surface for similar grid resolution in the surfacenormal direction. At $K n=0.03$ considerable efficiency gains are found in UFS NS calculations relative to LeMANS, presumably due to a larger difference in cell count between UFS and LeMANS at this higher Knudsen number value.

\section{Conclusions}

Results have been presented from a series of simulations for hardsphere monatomic gas flows over a cylinder at a freestream Mach number of 4 . A wide range of global Knudsen numbers have been considered, including one case $(K n=0.3)$ involving strong nonequilibrium over nearly the entire flowfield, a second case $(K n=0.03)$ involving significant regions of both continuum and rarefied flow, and a third case $(K n=0.003)$ for which the NS equations are valid over nearly the full simulation domain. These flow problems have been used to evaluate various models and combinations of models in the UFS code and to provide insight on the source of discrepancies found in previous work. Techniques employed in UFS code hold considerable promise for simulating a range of hypersonic gas-flow problems, particularly flows involving strong two-way coupling between rarefied and continuum regions, unsteady multiscale flows with a wide range of local Knudsen number regimes, and other high Knudsen number flows for which the inherent statistical scatter in DSMC is unacceptable or problematic.

As expected, Boltzmann simulation results for the highest Knudsen number case showed excellent overall agreement with DSMC. Large efficiency gains were realized when BGK calculations were used in place of Boltzmann calculations, although BGK simulations were found to give reduced accuracy relative to Boltzmann or DSMC simulations. Much of the error in BGK results is presumably due to underlying approximations in the BGK equation. Additional errors were found in surface quantities computed using either BGK or Boltzmann solvers; these errors are most likely associated with gradient approximations involving cut-cell boundaries and should be reduced or eliminated through implementation of an immersed boundary method in UFS.

A hybrid BGK-NS simulation performed for the $K n=0.03$ case showed good overall accuracy, but only small efficiency improvements were demonstrated for this simulation relative to corresponding BGK and DSMC simulations. The added complexity of a hybrid BGK-NS technique may therefore not be warranted for this case. Significantly reduced accuracy was found in a hybrid BGKEuler simulation for the $K n=0.03$ case, demonstrating the importance of diffusive transport effects within continuum regions of this flow. Improved agreement between BGK-Euler and DSMC results is expected if a more conservative continuum breakdown criterion is employed, but results seem to indicate the importance of accounting for viscous effects throughout the simulated flowfield. 
An important characteristic demonstrated in this study is the enormous computational expense of the discrete velocity Boltzmann solver in relation to DSMC. The UFS Boltzmann simulation for the $K n=0.3$ case was over two orders of magnitude more expensive than the corresponding DSMC simulation. Although computational expense may not be a driving concern for this relatively simple type of problem, the large discrepancy in simulation efficiency implies that, for a given set of computational resources, a DSMC code should be capable of simulating certain more complex rarefied gas flows that would be prohibitively expensive to simulate using the Boltzmann solver in UFS.

The poor relative efficiency of Boltzmann calculations can be primarily attributed to the large number of simulated binary interactions during each iteration, as required for sufficiently low scatter in simulation results. Although DSMC calculations also involve binary collision operations, several important differences exist between DSMC and discrete velocity Boltzmann solvers in both the procedures and characteristics of collision modeling: Most importantly, DSMC particles typically participate in no more than one collision per time step, whereas each velocity grid node in a Boltzmann simulation may be influenced by a large number of binary interactions during each step. This allows for potentially very low scatter in a Boltzmann calculation involving a relatively small number of velocity grid nodes, in contrast to the large expected scatter for a DSMC calculation involving a small number of particles per cell. Although DSMC calculations can achieve arbitrarily low scatter by means of very large particle populations, the additional memory requirements, and the additional computational expense associated with particle movement and cell indexing, generally make the use of large DSMC particle populations impractical as a way to match typical scatter levels in a Boltzmann simulation.

In a second difference between DSMC and Boltzmann collision operations, each binary collision in a Boltzmann calculation influences at least four velocity grid nodes (i.e., two incident velocities and at least two reflected velocities), whereas DSMC binary collisions involve no more than two particles. Additional operations are often required as part of Boltzmann collision procedures in order to enforce conservation of mass, momentum and energy. Third, for faster statistical convergence, Boltzmann collision calculations (including those in UFS) may employ low-discrepancy sequences in place of calls to a random number generator. Fourth, at a given point in velocity space, the frequency of participation in simulated collisions is typically independent of probability density in a Boltzmann simulation but not in DSMC; this tends to result in disproportionately high relative scatter in the tails of a DSMC distribution function. Other differences between DSMC and Boltzmann calculations include the lack of velocity discretization errors in DSMC and the need to define a finite velocity space domain for a Boltzmann simulation.

In comparing attributes and deficiencies between DSMC and the UFS Boltzmann solver, it should be noted that direct numerical simulation of the Boltzmann equation has several advantages over DSMC that in some cases may outweigh any disadvantages in efficiency. In particular, unlike DSMC or hybrid DSMC-CFD techniques, the UFS Boltzmann solver avoids complications associated with large DSMC statistical scatter, with no need for time-averaged sampling and no loss in precision if calculations are immediately stopped once steady-state conditions have been reached. As discussed in the introduction, low scatter is cited as the main reason for employing a Boltzmann solver in UFS, for strongly coupled hybrid calculations of mixed rarefied/continuum gas flows.

\section{Acknowledgments}

The authors gratefully acknowledge the Michigan/AFRL/Boeing Collaborative Center in Aeronautical Sciences, which provides funding for the work presented in this paper. The authors would also like to thank Robert Arslanbekov and Vladimir Kolobov for several helpful discussions and insightful suggestions over the course of this work. Additional assistance and helpful suggestions were provided by Ashley Verhoff.

\section{References}

[1] Bird, G. A., Molecular Gas Dynamics and the Direct Simulation of Gas Flows, Clarendon Press, Oxford, 1994.

[2] Vincenti, W. G., and Kruger, C. H., Introduction to Physical Gas Dynamics, Krieger, Malabar, FL, 1986.

[3] Schwartzentruber, T. E., and Boyd, I. D., "A Modular ParticleContinuum Numerical Method for Hypersonic Non-Equilibrium Gas Flows," Journal of Computational Physics, Vol. 225, 2007, pp. 1159 1174.

doi:10.1016/j.jcp.2007.01.022

[4] Wu, J.-S., Lian, Y.-Y., Cheng, G., Koomullil, R. P., and Tseng, K.-C., "Development and Verification of a Coupled DSMC-NS Scheme Using Unstructured Mesh," Journal of Computational Physics, Vol. 219, 2006, pp. 579-607.

doi:10.1016/j.jcp.2006.04.013

[5] Kaplan, C. R., Liu, J., and Oran, E. S., "Parallel Hybrid Method for Subsonic Flows: Coupling and Load-Balancing Challenges," AIAA Paper 2006-992, 2006.

[6] Garcia, A. L., Bell, J. B., Crutchfield, W. Y., and Alder, B. J., “Adaptive Mesh and Algorithm Refinement Using Direct Simulation Monte Carlo," Journal of Computational Physics, Vol. 154, 1999, pp. $134-155$.

doi:10.1006/jcph.1999.6305

[7] Wadsworth, D. C., and Erwin, D. A., "Two-Dimensional Hybrid Continuum/Particle Approach for Rarefied Flows," AIAA Paper 922975, 1992.

[8] Hash, D. B., and Hassan, H. A., "Assessment of Schemes for Coupling Monte Carlo and Navier-Stokes Solution Methods," Journal of Thermophysics and Heat Transfer, Vol. 10, No. 2, 1996, pp. 242-249. doi: $10.2514 / 3.781$

[9] Xu, K., and Huang, J.-C., "A Unified Gas-kinetic Scheme for Continuum and Rarefied Flows," Journal of Computational Physics, Vol. 229, 2010, pp. 7747-7764. doi:10.1016/j.jcp.2010.06.032

[10] Burt, J. M., and Boyd, I. D., "Extension of a Multiscale Particle Scheme to Near-Equilibrium Viscous Flows," AIAA Journal, Vol. 47, No. 6, 2009, pp. 1507-1517. doi: $10.2514 / 1.40262$

[11] Burt, J. M., and Boyd, I. D., "A Hybrid Particle Scheme for Simulating Multiscale Gas Flows with Internal Energy Nonequilibrium," AIAA Paper 2010-820, 2010.

[12] Kolobov, V. I., Arslanbekov, R. R., Aristov, V. V., Frolova, A. A., and Zabelok, S. A., "Unified Solver for Rarefied and Continuum Flows with Adaptive Mesh and Algorithm Refinement," Journal of Computational Physics, Vol. 223, 2007, pp. 589-608. doi:10.1016/j.jcp.2006.09.021

[13] Kolobov, V. I., Bayyuk, S. A., Arslanbekov, R. R., Aristov, V. V., Frolova, A. A., and Zabelok, S. A., "Construction of a Unified Continuum/Kinetic Solver for Aerodynamic Problems," Journal of Spacecraft and Rockets, Vol. 42, No. 4, 2005, pp. 598-606. doi:10.2514/1.10468

[14] Josyula, E., Arslanbekov, R. R., Kolobov, V. I., and Gimelshein, S. F., "Evaluation of Kinetic/Continuum Solver for Hypersonic NozzlePlume Flow," Journal of Spacecraft and Rockets, Vol. 45, No. 4, 2008 , pp. $665-676$. doi:10.2514/1.35431

[15] Arslanbekov, R. R., Kolobov, V. I., Frolova, A., Zabelok, S., and Josyula, E., "Evaluation of a Unified Kinetic/Continuum Solver for Computing Heat Flux in Hypersonic Blunt Body Flows," AIAA Paper 2007-4544, 2007.

[16] Dinavahi, S., and Josyula, E., "Simulation of Mach 3 Cylinder Flow Using Kinetic and Continuum Solvers," Department of Defense HPCMP User Group Conference, San Diego, CA, 2009.

[17] Popinet, S., "Gerris: A Tree-Based Adaptive Solver for the Incompressible Euler Equations in Complex Geometries," Journal of Computational Physics, Vol. 190, 2003, pp. 572-600. doi:10.1016/S0021-9991(03)00298-5

[18] Xu, K., "A Gas-Kinetic BGK Scheme for the Navier-Stokes Equations and its Connection with Artificial Dissipation and Godunov Method," Journal of Computational Physics, Vol. 171, 2001, pp. 289-335. doi:10.1006/jcph.2001.6790

[19] Dietrich, S., and Boyd, I. D., "Scalar and Parallel Optimized Implementation of the Direct Simulation Monte Carlo Method," Journal of Computational Physics, Vol. 126, 1996, pp. 328-342. doi:10.1006/jcph.1996.0141

[20] Scalabrin, L. C., and Boyd, I. D., "Development of an Unstructured Navier-Stokes Solver for Hypersonic Nonequilibrium 
Aerothermodynamics," AIAA Paper 2005-5203, 2005.

[21] Laney, C. B., Computational Gasdynamics, Cambridge Univ. Press, Cambridge, England, U.K., 1998.

[22] Wang, W., and Boyd, I. D., "Predicting Continuum Breakdown in Hypersonic Viscous Flows," Physics of Fluids, Vol. 15, No. 1, 2003, pp. $91-100$.

doi: $10.1063 / 1.1524183$

[23] Lofthouse, A. J., "Hypersonic Blunt Body Thermophysics Using a
Unified Kinetic/Continuum Solver," AIAA Paper 2009-3838, 2009.

[24] Maslach, G. J., and Schaaf, S. A., "Cylinder Drag in the Transition from Continuum to Free-Molecule Flow," Physics of Fluids, Vol. 6, No. 3 , 1963, pp. 315-321. doi:10.1063/1.1706736

[25] Josyula, E., Xu, K., Suchyta, C. J., and Bailey, W. F., "Kinetic Methods for Solving the Internal Structure of Shock Waves," AIAA Paper 20093841,2009 . 